\title{
Self-organized Notch dynamics generate stereotyped sensory organ patterns in Drosophila
}

Francis Corson $^{1 \dagger}$, Lydie Couturier ${ }^{2,3}$, Hervé Rouault ${ }^{2,3,4}$, Khalil Mazouni ${ }^{2,3}$ and François Schweisguth ${ }^{2,3 \dagger}$

1. Laboratoire de Physique Statistique, Ecole Normale Supérieure, CNRS, Université Pierre et Marie Curie, Université Paris Diderot, 75005 Paris, France

2. Institut Pasteur, Dept of Developmental and Stem Cell Biology, 75015 Paris, France

3. CNRS, UMR3738, 75015 Paris, France

4. present address: HHMI, Janelia Farm, Ashburn, VA 20147, USA

$\dagger$ co-corresponding authors: corson@lps.ens.fr; fschweis@pasteur.fr

One Sentence Summary: Distributed and flexible patterning combines with cell-cell interactions to set the rows of sensory hairs in order.

\begin{abstract}
:
The emergence of spatial patterns in developing multicellular organisms relies on positional cues and cell-cell communication. Drosophila sensory organs have informed a paradigm where these operate in two distinct steps: prepattern factors drive localized proneural activity, then Notchmediated lateral inhibition singles out neural precursors. Here we show that self-organization through Notch signaling also organizes the proneural stripes that resolve into rows of sensory bristles on the fly thorax. Patterning, initiated by a gradient of Delta ligand expression, progresses through inhibitory signaling between and within stripes. Thus Notch signaling can support selforganized tissue patterning as a prepattern is transduced by cell-cell interactions into a refined arrangement of cellular fates.
\end{abstract}

\section{Main text:}

Developmental patterning relies on morphogens ( 1 ) and cell-cell interactions (2) to define pattern formation. Sensory bristles on the Drosophila epidermis (3-6) derive from precursor cells (sensory organ precursors, SOPs) that themselves emerge from groups of cells expressing proneural genes of the Achaete-Scute Complex $(A S-C)$. SOPs are selected through Notchmediated lateral inhibition. Bristle patterns reflect the regulation of cell potential by prepattern factors (7); the pattern of large bristles (macrochaetae) depends on prepattern factors acting on individual enhancers in the $A S-C$, which drive expression in the corresponding proneural clusters (8). In contrast, patterning of the finer bristles (microchaetae; fig. S1, A and B) does not seem to involve stripe-specific enhancers (fig. S1C). We asked whether Notch - in addition to its role in SOP selection within proneural stripes - might also organize the sequential emergence of the stripes themselves. 


\section{Patterning dynamics}

To study the dynamics of Notch signaling and fate specification in the notum, we developed functional fluorescently tagged versions of the proneural proteins Achaete (Ac) and Scute (Sc), the Notch ligand Delta (Dl) and E(spl)m3-HLH, a direct transcriptional target of Notch, using BAC transgenes and CRISPR-mediated Homologous Recombination (HR). We used these reporters as well as antibodies against endogenous Dl and Senseless (Sens, a SOP marker) to study their expression patterns at successive time points during pupal development (Fig. 1, fig. S2, and fig. S3).

Analysis of early pupae identified two phases in the development of the pattern as the two heminotum epithelia fuse and move dorsally following head eversion. Up to 5-6 $\mathrm{h}$ after puparium formation (APF), the pattern of signaling activity was essentially stationary, and proneural proteins were confined to macrochaeta positions. In the next 6 to 7 hours, the microchaeta pattern was established. Throughout these two phases, notum cells are blocked in G2 and do not divide.

Before 5-6 h APF, expression of Dl exhibited a smoothly varying pattern, peaking at locations of future proneural stripes 1 and 5, with minimal levels where proneural stripe 3 will emerge. We observed Notch activity (expression of E(spl)m3-HLH as monitored by GFP-m3) only where D1 activity was intermediate, on the sides of the two Dl stripes. Thus, Notch activity formed a negative template for stripes 1,3 , and 5 (Fig. 1B).

After 5-6 h APF, Ac and Sc expression emerged in stripes 1, 3, and 5 (Fig. 1, C and D), accompanied by Dl expression in stripe 3 (fig. S3). By $7 \mathrm{~h}$ APF, these first three proneural stripes were being refined, with a subset of cells expressing Ac, Sc, and/or Sens, while the remaining cells expressed E(spl)m3-HLH (Fig. 1E and fig. S2). Concomitantly, the D1 stripes became narrower (fig. S3), and the range of Notch activity along stripes 1 and 5 decreased, giving rise to two bands of low Notch activity in the region of stripes 2 and 4 (Fig. 1E and fig. S2, B and C). By $8 \mathrm{~h}$ APF, expression of proneural genes, as well as Dl, had emerged in these gaps, completing the pattern of proneural stripes (Fig. 1F and fig. S3). Subsequently, the stripes resolved into SOPs; by 12 h APF, expression of Sens was restricted to regularly spaced cells in each row, with high levels of E(spl)m3-HLH in the remainder of the tissue (Fig. 1G). Further refinement of the pattern through addition or loss of SOPs and cell movements (9-11) is limited (fig. S4) and we concentrate on the period up to $12 \mathrm{~h}$ APF.

Live imaging of $\sim 6-10 \mathrm{~h}$ APF pupae confirmed the dynamics reconstructed from fixed samples. Although we were not able not follow patterning to its completion because the pupa moves at head eversion, we could observe the sequential emergence of proneural stripes and part of their resolution using GFP-Ac and GFP-Sc (movies S1 and S2). Imaging of D1-GFP and GPF-m3 showed the narrowing range of Dl expression and Notch activity in and along stripes 1 and 5, accompanied by the onset of signaling in stripe 3 (movies S3 and S4); at later stages, emerging SOPs were visible as isolated cells with low GFP-m3 levels within the stripes (movie S4).

These observations suggested the following patterning mechanism: a transient prepattern of Dl expression (independent from proneural gene expression) drives early Notch activity, providing a template for stripes 1,3 , and 5 , then the pattern self-organizes, with signaling from the firstformed providing a template for stripes 2 and 4, and signaling within stripes driving their resolution into SOP rows.

This model carries two implications. First, Dl and proneural gene expression must receive temporal inputs that delimit the two phases of patterning and control when the initial Dl gradient 
recedes and when the proneural genes are switched on. At $6 \mathrm{~h} \mathrm{APF,} \mathrm{Sc} \mathrm{expression} \mathrm{was} \mathrm{stronger} \mathrm{in}$ stripe 5 than in stripes 1 and 3, and fewer cells expressed Ac than Sc in stripe 3 (fig. S2A), suggesting slight differences in the timing of activation between Ac and Sc and across the tissue. Second, cells in the middle of the initial Dl stripes must be irresponsive to the high levels of Dl exposed by their neighbors to yield the observed pattern of Notch activity (Fig. 1B). Hypothesizing that this could result from cis-inhibition of the Notch receptor by $\mathrm{Dl}(12,13)$, we generated mosaic pupae carrying $\mathrm{Dl}$ mutant clones. Indeed, ectopic Notch activation in mutant cells along clone borders (Fig. 1H) indicated that Notch is cis-inhibited by Dl in the region of stripes 1 and 5.

\section{A mathematical model}

To further explore a self-organized patterning mechanism, we formulated a mathematical model of Notch signaling and fate specification (Fig. 2). The state of each cell is described by a single variable $u$, which takes values between 0 (low proneural activity / epidermal fate) and 1 (high proneural activity / SOP fate). A cell produces an inhibitory signal $D^{*}(u)$, representing its level of active Delta ligand, and receives a signal $s$ that sums contributions from neighboring cells,

$$
S_{i}=c_{i j} D *\left(u_{j}\right)
$$

where $c_{i j}$ describes the distance-dependent coupling between cells $i$ and $j$. In these terms, a generic model for signal-dependent fate specification (14) takes the form of a differential equation for the evolution of cell state,

$$
\frac{d u}{d t}=f(u, s) \quad u+(t)
$$

where the stochastic term $\eta(t)$ allows for fluctuations in signaling and gene expression. The dynamics of a cell can be represented as downhill motion in a one-dimensional landscape that shifts in response to signaling, and summarized in a bifurcation diagram (steady states vs. signal level) (Fig. 2A).

Based on a qualitative analysis of patterning dynamics, the model satisfied the following constraints (Fig. 2, A, C, and D): (i) inhibitory signaling from a cell increases faster than linearly as it progresses towards the SOP fate; (ii) the range of signaling exceeds immediate neighbors; (iii) in the absence of signal, a cell adopts the SOP fate; (iv) under high enough signal, it adopts the epidermal fate; (v) under intermediate signal levels, cells exhibit a bistable response - both fates are accessible. Our choice of

$$
f(u, s) \quad f\left(\begin{array}{ll}
u & s
\end{array}\right)
$$

with $f$ going from 0 to 1 as a sigmoid (Fig. 2B), balances inhibition and positive feedback on $u$, required for bistability. Although the variable $u$ is not intended to represent the level of an individual protein, Eqs. 2 and 3 are formally equivalent to a phenomenological model for gene regulation (15), with a linear regulatory input $(u-s)$, a saturating response $(f)$, and a linear degradation term $(-u)$.

To simulate patterning, we prescribed a time-dependent gradient of inhibitory signal that mimicked Notch activity around stripes 1 and 5. As the gradient narrowed down, the model 
recapitulated the sequential emergence of stripes 2-4 and their resolution into SOP rows (Fig. 2E). To also describe the emergence of stripes 1 and 5, we incorporated cis-inhibition in the model (supplementary text S1). With cis-inhibition, specifying a transient gradient of D1 expression recapitulated the full sequence of five proneural stripes and SOP rows (fig. S5). However, because cis-inhibition is not essential for patterning, we concentrate on the simpler model.

\section{Attrition of the proneural group and cell-intrinsic bistability}

Expression patterns in fixed samples implied a progressive refinement of proneural stripes, extending over several hours. Stripe 3, which was formed by $6 \mathrm{~h}$ APF and already being refined at $7 \mathrm{~h} \mathrm{APF}$, was not fully resolved at $8 \mathrm{~h}$ APF (Fig. 1, D to G, fig. S2, A and B, and fig. S6A). Likewise, Sens was expressed in continuous bands of cells in emerging stripes 2 and 4 at $8 \mathrm{~h}$ APF (Fig. 3A); adjacent Sens-expressing cells remained at $10 \mathrm{~h} \mathrm{APF} \mathrm{(Fig.} \mathrm{3A)} \mathrm{but} \mathrm{not} 12 \mathrm{~h} \mathrm{APF} \mathrm{(Fig.}$ $1 \mathrm{G}$ and fig. S4). At intermediate stages of stripe resolution, cells expressing Ac, Sc, and/or Sens were often arranged in nearly continuous rows, suggesting that resolving stripes initially narrow down. Live imaging of GFP-Sc supported these observations (Fig. 3B and movie S2).

In spite of qualitative similarities, Ac, Sc, and Sens exhibited different expression patterns, hinting to differences in their dynamics. At $8 \mathrm{~h}$ APF, Ac was expressed broadly in emerging stripes, while Sc and Sens were restricted to cells with high Ac (figs. S2A and S6). Sc and Sens, but not Ac, reached higher levels in resolving stripes than emerging stripes. This suggested that Ac plateaus in a broad proneural group, while Sc and Sens levels increase in cells progressing towards the SOP fate (thus Sc provides a better readout of SOP selection dynamics than Ac; compare movies S1 and S2). Fewer cells expressed Sens than Sc in resolving stripes, suggesting that Sens is downregulated more rapidly in cells that are excluded from the proneural group.

Our observations indicated that proneural stripes are initially refined through exclusion of lateral cells. While a persistent spatial bias extrinsic to the stripe could favor more central cells, the pattern of Notch activity argued against this possibility. Indeed, at $7 \mathrm{~h}$ APF Notch was activated on the sides of stripe 3, while signaling activity had receded in the regions of stripe 2 and 4 (Fig. $1 \mathrm{E}$ and fig. S2, B and C). Thus, exclusion of cells on the sides of stripe 3 must result from mutual inhibition within stripe 3, implying that stripe 3 narrows down autonomously. However, cells on the sides are likely exposed to lower Dl levels than cells at the center of the stripe. This apparent paradox is resolved if cells exhibit a bistable response, as required in our model: central cells, which are more advanced towards the SOP fate, can keep progressing under signal levels that are sufficient to inhibit lateral cells.

Specifically, the model suggests the following scenario for stripe resolution (Fig. 4, A to C). The onset of proneural activity in an extended group of cells is accompanied by weak mutual inhibition. Idealizing the proneural group as equivalent cells in a state $u$, each cell receives a signal $s=C_{0} D^{*}(u)$, where $C_{0}$ is the effective number of neighbors of a cell, $C_{0} \approx 18$ with our parameters for signaling. Under mutual inhibition, perfectly equivalent cells would tend to a uniform steady state with low $u$. However, this steady state has each cell poised at the tipping point between the two fates, making it unstable regardless of the strength of mutual inhibition (supplementary text S3). Thus, cells are necessarily removed from the proneural group, allowing further progression of the remaining cells. Idealizing an intermediate state of stripe resolution as a file of equivalent cells, mutual inhibition with fewer effective neighbors, $C_{1} \approx 3$, leads to a steady state with a higher value of $u$, which again is unstable. Finally, a pair of adjacent cells with high $u$ 
is unstable, guaranteeing that only isolated SOPs remain in the final pattern. Thus, the qualitative structure of the model ensures a gradual attrition of the proneural group. In the absence of spatial bias, which cells are excluded first depends on noise, resulting in a disordered pattern. However, since emerging proneural stripes are delimited by a gradient of inhibitory signaling, lateral cells are biased for early exclusion (Fig. 4D), and stripes narrow down before resolving.

Because the model introduces no extrinsic bias along the A-P axis, symmetry breaking in that direction depends on the amplification of random differences. With a broad range of unstable modes (supplementary text S3), intermediates states are variable in structure; pairs of cells with high $u$ can persist for some time (Fig. 2E, $t=2$, movie S5). As attrition proceeds, however, a regular spacing emerges: SOPs must be distant enough to evade each other's inhibition, yet not too distant or intervening cells would not be inhibited.

Our experimental observations, including early exclusion of lateral cells (Fig. 1E), restriction of proneural activity to central cells (Fig. 3) and persistent expression of Sens in cell pairs (e.g. in stripes 2 and 4 at $10 \mathrm{~h} \mathrm{APF,} \mathrm{Fig.} \mathrm{3A),} \mathrm{supported} \mathrm{this} \mathrm{scenario.} \mathrm{As} \mathrm{a} \mathrm{signature,} \mathrm{time} \mathrm{courses} \mathrm{of} \mathrm{cell}$ state in the model showed a gradual progression of cells towards the SOP fate, accompanied by a continuous branching off of cells that reverted to low $u$ (Fig. 5, A and B). When we quantified Sc levels in individual cells in vivo, we observed a similar structure (Fig. 5, C and D). In both model and experiments, cells at intermediate positions between two SOPs could progress for an extended period of time and reach high levels of $u$ or Sc before eventually being inhibited.

As a counterpoint, we considered a model lacking cell-intrinsic feedback, i.e. $f(u, s) \equiv f(s)$ in Eq. 2 (supplementary text S2). Compared to the bistable case, patterning in a monostable model required stronger non-linearities, e.g. a sharp step in the signal-sending curve $D^{*}(u)$ (figs. S7 and S8). While simulations showed that a monostable model could produce a pattern of SOP rows, these did not emerge through a gradual narrowing of the proneural group. Instead, cells within a stripe first plateaued at intermediate values of $u$, then isolated SOPs emerged, while the remaining cells reverted to low $u$ (fig. S8, B and C). A monostable response also allowed cells to remain at intermediate levels of $u$ at steady state (fig. S8, B and C), when bistability enforces a clear segregation between cells with high and low $u$. Based on a linear stability analysis (supplementary text S3), a bistable model supports the emergence of a large-scale structure (on the scale of the signaling range, like proneural stripes); in a monostable model, this is suppressed and instability is driven by small-scale fluctuations (fig. S9).

\section{Perturbation analysis}

Patterning in the model depends on defined boundary conditions and signaling dynamics, and is predicted to break down in different ways when these are affected.

Self-organized proneural stripes should be displaced when Notch activity is perturbed in the neighboring tissue. Indeed, ectopic Notch mutant SOPs created gaps in the proneural stripes in the surrounding wild-type tissue (Fig. 6, A and A').

Proneural stripes must be narrow enough, relative to the range of inhibition, to resolve into a central SOP row. Reduced Dl levels, affecting early Notch activity, should result in a broader stripe 3 , and also weaken lateral inhibition within the stripes. In the model, reducing Dl levels by a half was sufficient to disrupt patterning. As predicted, a disordered pattern developed in the interval between rows 1 and 5 in $D l$ heterozygous pupae (Fig. 6, B and B'). 
By contrast, mutations affecting scabrous (sca) reduce the range of lateral inhibition (16), but should leave early patterns unaffected because sca is only expressed in proneural cells (17). With a moderate reduction in signaling range, the model predicted a normal stripe 3, but stripes 2 and 4 split to form supernumerary rows (Fig. 6C and fig. S10B). In sca mutant pupae, we observed expression patterns that were hardly distinguishable from the wild type up to $8 \mathrm{~h} \mathrm{APF}$, but more densely packed SOPs at later stages (fig. S10A); the arrangement of these SOPs suggested supernumerary rows. When we observed Sc expression in vivo in sca mutants, we could observe the splitting of proneural stripes, as predicted (Fig. 6C').

Our model requires a nonlinear increase in signaling from emerging SOPs. If $D^{*}(u)$ varies linearly with $u$, either the stripes fail to emerge, because mutual inhibition is too strong, or they fail to resolve, because inhibition is too weak (fig. S11). Dl is broadly expressed within emerging proneural stripes (fig. S3), arguing against a sharp upregulation of its expression in SOPs. Instead, differences in Dl activity levels may rely on post-translational regulation. The E3 ubiquitin ligase Neuralized (Neur) is a key regulator of Dl activity that is specifically expressed in emerging SOPs $(18-20)$. In our model, this is represented as $D^{*}(u)=a(u) D(u)$, where the Delta level $D(u)=u$ varies linearly as a default, and the ligand activity $a(u)$ increases from a basal level $a(0)=a_{0} \square 1$ to $\mathrm{a}(1)=1$ upon full activation. When we simulated patterning with reduced or no activity enhancement, stripes formed normally but failed to resolve (Fig. 6D and fig. S12). Such a phenotype is observed within neur mutant clones (21) (Fig. 6D') and in gain-of-function mutants of Bearded (22), an inhibitor of Neur (23). In terms of our model, increased SOP density in stripes 1, 3, and 5 maintains an inhibitory template for stripes 2 and 4.

The model can also account for the loss or gain of bristle rows in flies that are smaller or larger than average (fig. S13), an observation that hinted to a dynamic origin of proneural stripes (24). Together, these perturbation experiments provide strong support for the model.

\section{Discussion}

Proneural patterning is classically viewed as an output of positional information that is interpreted by specific cis-regulatory enhancers at proneural loci (4) or relayed via proneural inhibitors (25). Proneural patterning has also been proposed to depend on Notch. In the inner ear of vertebrates, Notch relays positional information to maintain undifferentiated progenitor cells (26). Whether Notch specifies, rather than maintains, prosensory domains remains, however, controversial (26-28). Similarly, Notch has been proposed previously to pattern proneural stripes (29). However, the origin of the Notch activity pattern was not addressed. Here, time-resolved analysis of patterning dynamics establishes that both positional cues (setting up the initial pattern of $\mathrm{Dl}$ ) and downstream cell-cell interactions contribute to pattern Notch activity, which in turns acts as a negative template for proneural gene expression. Thus, prepattern and self-organization, operating through the same Notch signal, combine during bristle development. While stripes 1 and 5 play equivalent roles in our model, we observed minor differences. For instance, stripe 1 resolves later and SOPs emerge along the distal side of stripe 1. Thus, other regulatory inputs, e.g. signals produced by midline cells, may impinge on patterning.

While the relevant cellular and molecular interactions are well characterized, the mechanism by which neural precursors are selected among proneural cells has remained controversial (3). In many accounts, proneural clusters either comprise a small number of cells, such that competition between contacting cells is sufficient to select isolated precursors (30), or a subgroup of more competent cells is delimited by an inhibitory template (31). In our model, the restriction of 
competence is dynamic and driven by mutual inhibition: cells are continuously excluded as the level of inhibition increases. Signaling modulators, e.g. Neur, likely drive the gradual transition from weak mutual inhibition within an extended proneural group to strong lateral inhibition from emerging neural precursors.

Patterning occurs in a two-dimensional field of non-dividing cells, prior to the first wave of cell division (32). There are often 3-4 epidermal cells between SOPs within rows, and a comparable or larger interval is seen between rows, while Notch is activated in all non-SOP cells. As noted previously $(11,16,33)$, this implies that the range of signaling must exceed immediate neighbors. Since Notch signaling requires direct cell-cell contact, it could be mediated via basal filopodia that connect distant cells $(11,16,33)$.

Our model represents proneural activity by a single variable $u$ that subsumes the dynamics of multiple genes, e.g. Ac, Sc, and Sens. Likewise, Dl activity is an instantaneous function of cell state. These simplifying assumptions, which allow a systematic analysis, could be rationalized by a model where different genes act as different readouts of a common regulatory input, integrating positive feedback and inhibition. In these terms, broad expression of Ac in an extended proneural group hints to a more threshold-like response than Sc and Sens patterns, which are more graded in space and time. Earlier resolution of Sens patterns, compared to Ac and Sc, is consistent with Sens acting as a switch in SOP selection $(34,35)$; proneural activity may gradually recede following Sens downregulation.

Most previous models of lateral inhibition have focused on the dynamics of Dl and Notch (36), in the absence of cell-intrinsic feedback. Provided that mutual interactions are sufficiently strong, adjacent cells with high Dl levels cannot coexist, and initially equivalent cells resolve into a saltand-pepper pattern $(36)$. On the other hand, several recent models $(37,38)$ have incorporated cellintrinsic bistability, which allows emergent neural precursors to evade inhibition from their neighbors; in the presence of an extrinsic bias, this makes it possible for cells at the center of a proneural cluster to be selected (38). Dependent on particular parameter choices, these studies $(37,38)$ focused on a regime where cells crossing a threshold in the progression to the neural fate become refractory to inhibition. In this regime, adjacent neural precursors can coexist, and the selection of isolated cells depends on isolated cells crossing the threshold. Both studies $(37,38)$ suggested a requirement for a sharp onset of inhibitory signaling. Then, signaling is mostly in the form of lateral inhibition from committed neural precursors, and all neighboring cells are excluded within a short time interval, compared with the time needed to approach the threshold, i.e. an order of magnitude shorter (38). In that limit, precursor selection can be described as a "race for the neural fate" (38). The model introduced here, with different choices of the response function $f$, can recover these two limiting behaviors in a compact form (one variable per cell), and it allowed us to explore an intermediate regime, where cells are intrinsically bistable, yet remain responsive to inhibition. This regime combines the desirable features that neural precursors emerge from the center of the proneural group and adjacent precursors are excluded. In addition, precursors emerge through a gradual narrowing of the proneural group, with central cells first inhibiting more lateral cells, then "competing" among themselves to become SOPs. These properties mesh well with our observations in the notum, showing an early onset of mutual inhibition within stripes, well before SOPs emerge, and progressive refinement of the proneural group, throughout the progression of the eventual SOPs.

Mechanistically, bistability could result from positive feedback on proneural activity $(38,39)$ and/or cis-inhibition of Notch $(37,40)$. While our main model abstracts the consequences of 
bistability from its molecular basis, we also considered a model that explicitly incorporates cisinhibition. In this model, we prescribed Dl levels that were sufficient to titrate Notch within stripes 1 and 5, but not in SOPs, which remained responsive to inhibition. The extent to which Dl cis-inhibits Notch in SOPs remains to be experimentally tested.

Notch mediates patterning in other epithelia in flies as well as in other species (41-46). In the Drosophila eye, expression of the proneural gene Atonal begins as a broad stripe and becomes restricted to isolated R8 photoreceptors (41). Stripes of Dl expression also pattern bristle rows in the Drosophila leg (42). The interplay between cell-intrinsic bistability and cell-cell interactions may provide a simple framework to explain how distinct patterns are produced by varying boundary conditions.

\section{Materials and methods:}

\section{Flies}

The $N^{55 e 11}, s c a^{B P 2}, s c a^{1}, D l^{R e v 10}, \mathrm{Ser}^{R x 82}$, neur ${ }^{I F 65}$ mutations were used. FLP/FRT clones were detected using loss of nuclear RFP. GFP-Moe was expressed using neur-Gal4 (11).

The Delta ${ }^{G F P}$ and scute ${ }^{G F P}$ lines were generated using CRISPR-mediated HR. For each locus, two gRNA oligonucleotides were cloned into pU6-BbsI-chiRNA (Addgene \#45946) as described in addgene.org/crispr/OConnor-Giles/. Donor templates for HR were first produced by BAC recombineering in $E$. coli and then transferred into multicopy vectors as described in (47). BACs encoding $\mathrm{Dl}$ (48) and scute (BAC CH321-32O15) were used to introduce sfGFP flanked by GVG linkers, at the KGAS//GGPG position (Dl-PA) and at the N-terminus (Sc). The 3xP3-RFP selection marker flanked by loxP sites was produced by gene synthesis. Left and right homology arms flanking the target sites were $1.5 \mathrm{~kb}$ long. Proper HR was verified by genomic PCR. Delta $^{G F P}$ and scute ${ }^{G F P}$ flies were viable with no phenotype.

GFP-m3 was generated using recombineering mediated gap-repair (47) from the $E(s p l)-C$ BAC (49) and was integrated at the M\{3xP3-RFP.attP\}ZH-51D site. The GFP-Ac and Cherry6xMyc-Ac BACs were generated from the CH321-32O15 BAC that fully rescues the $s c^{10-1}$ mutation. BACs were integrated at the $\mathrm{PB}\{\mathrm{y}+$-attP3B $\}$ VK00033 (65B) landing site. Cloning details will be provided upon request.

\section{Immunostainings}

Staged pupae were dissected and stained following standard procedures. Primary antibodies were: rabbit and goat anti-GFP, rabbit anti-DsRed (Clonetech), guinea pig anti-Senseless (1:3000, kind gift from H. Bellen), rat anti-Delta (rat anti-DeltaICD, mAb 10D5, 1:1000, from M. Rand).

\section{Live imaging}

Staged pupae were mounted on a custom-made support and imaged through the pupal case. Movies were acquired using continuous imaging ( $\Delta \mathrm{t}=5-15 \mathrm{~min}$ ) with $\Delta \mathrm{z}=1.5 \mu \mathrm{m}$ using $\mathrm{z}$-stacks with 25-50 sections. A 63x (PL APO, N.A. 1.4 DIC M27) objective on a Zeiss LSM780 microscope was used. The Histone2Av-mRFP1 marker was used to detect all nuclei. All times are equivalent hours at the reference temperature of $25^{\circ} \mathrm{C}$. 


\section{Image processing}

Images were processed using custom Java software built on the ImageJ API.

Projections of live images were generated after subtraction of signal from the cuticle. The location of the cuticle, which produced a stronger signal than nuclei, was determined by thresholding a smoothed image. To allow for spread in the $\mathrm{z}$ direction, the cuticle signal was extrapolated below the cuticle, assuming an exponential decay with z. After subtraction, a smooth surface was fit to the tissue, by minimizing a cost function that draws the surface towards areas of strong signal while penalizing sharp variations in its profile. Projections were obtained by summing pixel intensities in a small $\mathrm{z}$ range around the surface.

\section{Single-cell quantification}

Cell nuclei were identified using a variation on the watershed algorithm. To prevent oversegmentation due to inhomogeneous signal intensity, a binary mask was generated by thresholding a filtered image, then the watershed algorithm was applied to the distance transform of the mask. In the case of live images, individual z slices were segmented separately, then nuclei in consecutive slices were matched by proximity; together with a manual correction step, this allowed identification of nuclei when the pupa had moved between two slices. The level of signal in a nucleus was defined as the average intensity of its pixels. To compensate for variations in imaging conditions across the tissue, the GFP-Sc level obtained from live images was divided by the level of the nuclear marker, corrected for bleaching. Time courses were obtained by manual tracking of the nuclei.

\section{Mathematical model}

The dynamics of cell $i$ is described by a stochastic differential equation of the form

$$
\frac{d u_{i}}{d t}=f\left(u_{i}, s_{i}\right) \quad u_{i}+{ }_{i}(t)
$$

with a characteristic time scale $\tau$ and uncorrelated fluctuations

$$
\left\langle{ }_{i}(t)_{j}\left(t^{\prime}\right)\right\rangle=2 D^{2}{ }_{i j}\left(t \quad t^{\prime}\right)
$$

Our main model is obtained with

$$
f(u, s) \quad f\left(\begin{array}{ll}
u & s
\end{array}\right)=\left(2\left(\begin{array}{ll}
u & s
\end{array}\right)\right)
$$

where $\sigma$ is the sigmoidal function

$$
(x)=\frac{1+\tanh 2 x}{2}
$$

which goes from 0 to 1 with $\sigma(0)=1 / 2$ and $\sigma^{\prime}(0)=1$.

The signal $s_{i}$ received by a cell integrates a time-dependent gradient and signaling from neighboring cells 


$$
s_{i}=s_{j}\left(x_{i}, t\right)+{ }_{j} c_{i j} D *\left(u_{j}\right)
$$

where $x_{i}$ is the position of the cell along the medial-distal axis and

$$
c_{i j}=e^{\frac{d_{i j}^{2}}{2 l^{2}}}
$$

describes the coupling between cells $i$ and $j$ according to their distance $d_{i j}$, with the convention that $c_{i i}=0$.

The signal produced by a cell as a function of its state is

$$
D *(u)=a(u) D(u)
$$

where the ligand level $D(u)=u$ is taken to vary linearly as a default, and the ligand activity

$$
a(u)=a_{0}+\frac{3 u^{3}}{1+u^{2}} a_{1}
$$

increases from a basal level $a(0)=a_{0}$ to $a(1)=1$.

\section{Simulations of patterning}

Simulations were run on a fixed array of cells, occupying a unit square with periodic boundary conditions, such that stripes 1 and 5 are equivalent (as an exception, a larger box was used for Fig. S6A). Disordered cell arrangements were generated using a vertex model of epithelial tissue dynamics (50), with a random Voronoi tessellation as initial condition and a noise level $z=30 \%$ as per (50). The resulting cell arrangements had a majority of cells with six neighbors and a standard deviation of cell-cell distances of about $10 \%$ the average.

The signaling gradient

$$
S_{g}(x, t)=S_{0}\left(1 \frac{t}{g}\right)\left(e^{\frac{x^{2}}{2 L^{2}}}+e^{\frac{(1 x)^{2}}{2 L^{2}}}\right)+\left(\frac{t}{g}\right)\left(e^{\frac{x^{2}}{2 l^{2}}}+e^{\frac{(1 x)^{2}}{2 l^{2}}}\right)
$$

interpolated over a time scale $\tau_{g}$ between a Gaussian profile with peak level $S_{0}$ and width $L$, and a narrower profile representing signaling from SOPs in rows 1 and 5, with range $l$.

\section{Model parameters}

Parameter values (table S2) were chosen to reproduce wild-type patterning dynamics. A bistable response requires a slope $f^{\prime}>1$, and Eq. 6 was chosen to yield a slope of 2 as a default. The resulting model is bistable in a relatively narrow range of signal levels; with different choices of $f$ that yielded a broader bistable range, the emergence of stripes and/or their resolution were affected. The number $N$ of cells in simulations was chosen based on a typical cell-cell distance $\approx 5 \mu \mathrm{m}$ and distance between rows 1 and $5 \approx 75-100 \mu \mathrm{m}$ in experiments. The range $L$ of the initial gradient was adjusted to yield a stripe 3 that is a few cells wide. The signaling range $l$ had to be large enough for resolution of stripes into a central row of SOPs $(l=1.25 \lambda$ yields supernumerary rows, fig. S10B), but not too large or stripes 2 and 4 were suppressed (e.g. with 
$l=2.5 \lambda)$. Relative to the time scale of cell dynamics $(\tau)$, the decay of the initial gradient (parameterized by $\tau_{g}$ ) was chosen to be slow enough to pattern stripe 3 , but fast enough for stripes 2 and 4 to emerge before complete resolution of stripe 3, as observed in experiments (Fig. 1F). The shape of the activity curve $a(u)$ was chosen such that the steady states in Fig. 4B lie on and are spread across the unstable branch of the bifurcation diagram; this implied a basal activity $a_{0}$ of the order of $1 / C_{0}$. The noise level $D$ was large enough that different simulations with the same cell arrangement yielded different patterns (i.e. fluctuations primed over the frozen disorder in the cell arrangement), but small enough for orderly resolution of the pattern.

\section{References:}

1. L. Wolpert, Positional information and the spatial pattern of cellular differentiation. $J$ Theor Biol. 25, 1-47 (1969).

2. A. M. Turing, The chemical basis of morphogenesis. Philosophical Transactions of the Royal Society B: Biological Sciences. 237, 37-72 (1952).

3. L. I. Held, Imaginal Discs: The Genetic and Cellular Logic of Pattern Formation (Cambridge Univeristy Press, 2002).

4. J. L. Gómez-Skarmeta, S. Campuzano, J. Modolell, Half a century of neural prepatterning: the story of a few bristles and many genes. Nat Rev Neurosci. 4, 587-598 (2003).

5. P. Simpson, The stars and stripes of animal bodies: evolution of regulatory elements mediating pigment and bristle patterns in Drosophila. Trends in Genetics. 23, 350-358 (2007).

6. A. Garcia-Bellido, J. F. de Celis, The complex tale of the achaete-scute complex: a paradigmatic case in the analysis of gene organization and function during development. Genetics. 182, 631-639 (2009).

7. C. Stern, Two or three bristles. American Scientist. 42, 213-247 (1954).

8. J. L. Gomez-Skarmeta et al., Cis-regulation of achaete and scute: shared enhancer-like elements drive their coexpression in proneural clusters of the imaginal discs. Genes Dev. 9 , 1869-1882 (1995).

9. K. Usui, K.-I. Kimura, Sequential emergence of the evenly spaced microchaetes on the notum of Drosophila. Wilhelm Roux' Archiv. 203, 151-158 (1993).

10. O. Renaud, P. Simpson, Movement of bristle precursors contributes to the spacing pattern in Drosophila. Mech Dev. 119, 201-211 (2002).

11. M. Cohen, M. Georgiou, N. L. Stevenson, M. Miodownik, B. Baum, Dynamic filopodia transmit intermittent Delta-Notch signaling to drive pattern refinement during lateral inhibition. Dev Cell. 19, 78-89 (2010).

12. D. Sprinzak et al., Cis-interactions between Notch and Delta generate mutually exclusive 
signalling states. Nature. 465, 86-90 (2010).

13. D. del Álamo, H. Rouault, F. Schweisguth, Mechanism and significance of cis-inhibition in Notch signalling. Current Biology. 21, R40-R47 (2011).

14. F. Corson, E. D. Siggia, Geometry, epistasis, and developmental patterning. Proc Natl Acad Sci USA. 109, 5568-5575 (2012).

15. J. Reinitz, D. H. Sharp, Mechanism of eve stripe formation. Mech Dev. 49, 133-158 (1995).

16. O. Renaud, P. Simpson, scabrous modifies epithelial cell adhesion and extends the range of lateral signalling during development of the spaced bristle pattern in Drosophila. Dev Biol. 240, 361-376 (2001).

17. M. Mlodzik, N. E. Baker, G. M. Rubin, Isolation and expression of scabrous, a gene regulating neurogenesis in Drosophila. Genes Dev. 4, 1848-1861 (1990).

18. G. L. Boulianne, A. de la Concha, J. A. Campos-Ortega, L. Y. Jan, Y. N. Jan, The Drosophila neurogenic gene neuralized encodes a novel protein and is expressed in precursors of larval and adult neurons. EMBO J. 10, 2975-2983 (1991).

19. E. C. Lai, G. A. Deblandre, C. Kintner, G. M. Rubin, Drosophila neuralized is a ubiquitin ligase that promotes the internalization and degradation of Delta. Dev Cell. 1, 783-794 (2001).

20. E. Pavlopoulos et al., neuralized encodes a peripheral membrane protein involved in Delta signaling and endocytosis. Dev Cell. 1, 807-816 (2001).

21. R. Le Borgne, F. Schweisguth, Unequal segregation of Neuralized biases Notch activation during asymmetric cell division. Dev Cell. 5, 139-148 (2003).

22. M. W. Leviten, J. W. Posakony, Gain-of-function alleles of Bearded interfere with alternative cell fate decisions in Drosophila adult sensory organ development. Dev Biol. 176, 264-283 (1996).

23. A. J. Bardin, F. Schweisguth, Bearded family members inhibit Neuralized-mediated endocytosis and signaling activity of Delta in Drosophila. Dev Cell. 10, 245-255 (2006).

24. P. Simpson, R. Woehl, K. Usui, The development and evolution of bristle patterns in Diptera. Development. 126, 1349-1364 (1999).

25. A. Bhattacharya, N. E. Baker, A network of broadly expressed HLH genes regulates tissue-specific cell fates. Cell. 147, 881-892 (2011).

26. J. Neves, C. Parada, M. Chamizo, F. Giraldez, Jagged 1 regulates the restriction of Sox2 expression in the developing chicken inner ear: a mechanism for sensory organ specification. Development. 138, 735-744 (2011). 
27. B. H. Hartman, T. A. Reh, O. Bermingham-McDonogh, Notch signaling specifies prosensory domains via lateral induction in the developing mammalian inner ear. Proceedings of the National Academy of Sciences. 107, 15792-15797 (2010).

28. M. L. Basch, T. Ohyama, N. Segil, A. K. Groves, Canonical Notch signaling is not necessary for prosensory induction in the mouse cochlea: insights from a conditional mutant of RBPjkappa. Journal of Neuroscience. 31, 8046-8058 (2011).

29. A. L. Parks, S. S. Huppert, M. A. Muskavitch, The dynamics of neurogenic signalling underlying bristle development in Drosophila melanogaster. Mech Dev. 63, 61-74 (1997).

30. P. Heitzler, P. Heitzler, P. Simpson, P. Simpson, The choice of cell fate in the epidermis of Drosophila. Cell. 64, 1083-1092 (1991).

31. T. Troost, M. Schneider, T. Klein, A re-examination of the selection of the sensory organ precursor of the bristle sensilla of Drosophila melanogaster. PLoS Genet. 11, e1004911 (2015).

32. F. Bosveld et al., Mechanical control of morphogenesis by Fat/Dachsous/Four-jointed planar cell polarity pathway. Science. 336, 724-727 (2012).

33. C. De Joussineau et al., Delta-promoted filopodia mediate long-range lateral inhibition in Drosophila. Nature. 426, 555-559 (2003).

34. R. Nolo et al., Senseless, a Zn finger transcription factor, is necessary and sufficient for sensory organ development in Drosophila. Cell. 102, 349-362 (2000).

35. H. Jafar-Nejad, Senseless acts as a binary switch during sensory organ precursor selection. Genes Dev. 17, 2966-2978 (2003).

36. J. R. Collier, N. A. M. Monk, P. K. Maini, J. H. Lewis, Pattern formation by lateral inhibition with feedback: a mathematical model of Delta-Notch intercellular signalling. $J$ Theor Biol. 183, 429-446 (1996).

37. O. Barad, D. Rosin, E. Hornstein, N. Barkai, Error minimization in lateral inhibition circuits. Sci Signal. 3, ra51-ra51 (2010).

38. D. K. Lubensky, M. W. Pennington, B. I. Shraiman, N. E. Baker, A dynamical model of ommatidial crystal formation. Proceedings of the National Academy of Sciences. 108, 11145-11150 (2011).

39. J. Culí, J. Modolell, Proneural gene self-stimulation in neural precursors: an essential mechanism for sense organ development that is regulated by Notch signaling. Genes Dev. 12, 2036-2047 (1998).

40. D. Sprinzak, A. Lakhanpal, L. Lebon, J. Garcia-Ojalvo, M. B. Elowitz, Mutual inactivation of Notch receptors and ligands facilitates developmental patterning. PLoS Comput Biol. 7 , e1002069 (2011). 
41. J.-Y. Roignant, J. E. Treisman, Pattern formation in the Drosophila eye disc. Int. J. Dev. Biol. 53, 795-804 (2009).

42. M. Joshi, K. T. Buchanan, S. Shroff, T. V. Orenic, Delta and Hairy establish a periodic prepattern that positions sensory bristles in Drosophila legs. Dev Biol. 293, 64-76 (2006).

43. J. Lewis, Notch signalling and the control of cell fate choices in vertebrates. Semin Cell Dev Biol. 9, 583-589 (1998).

44. A. E. Kiernan, Notch signaling during cell fate determination in the inner ear. Semin Cell Dev Biol. 24, 470-479 (2013).

45. D. Henrique et al., Maintenance of neuroepithelial progenitor cells by Delta-Notch signalling in the embryonic chick retina. Curr Biol. 7, 661-670 (1997).

46. R. Sancho, C. A. Cremona, A. Behrens, Stem cell and progenitor fate in the mammalian intestine: Notch and lateral inhibition in homeostasis and disease. EMBO Rep. 16, 571-581 (2015).

47. K. J. T. Venken, Y. He, R. A. Hoskins, H. J. Bellen, P[acman]: a BAC transgenic platform for targeted insertion of large DNA fragments in D. melanogaster. Science. 314, 17471751 (2006).

48. L. Lebon, T. V. Lee, D. Sprinzak, H. Jafar-Nejad, M. B. Elowitz, Fringe proteins modulate Notch-ligand cis and trans interactions to specify signaling states. eLife. 3, e02950 (2014).

49. S. Chanet, N. Vodovar, V. Mayau, F. Schweisguth, Genome engineering-based analysis of Bearded family genes reveals both functional redundancy and a nonessential function in lateral inhibition in Drosophila. Genetics. 182, 1101-1108 (2009).

50. K. Sugimura, S. Ishihara, The mechanical anisotropy in a tissue promotes ordering in hexagonal cell packing. Development. 140, 4091-4101 (2013).

51. M. Cross, H. Greenside, Pattern Formation and Dynamics in Nonequilibrium Systems (Cambridge Univeristy Press, 2009).

52. E. Plahte, Pattern formation in discrete cell lattices. J Math Biol. 43, 411-445 (2001).

53. H. G. Othmer, L. E. Scriven, Instability and dynamic pattern in cellular networks. $J$ Theor Biol. 32, 507-537 (1971).

\section{Acknowledgments}

We thank B. Baum, H. Bellen, H. Jafar-Nejad, and M. Rand for reagents. We thank V. Roca for embryo injection. We also thank V. Hakim, B. Hassan, and E. Siggia for discussion and critical reading. Supported by ANR-16-CE13-0003 and ARC-PGA120140200771 grants. Supplement contains additional data. 


\section{Supplementary Materials}

Supplementary Text

Figs. S1 to S13

Tables S1 and S2

Movies S1 to S5

References (51-53)

\section{Figure legends:}

Fig. 1. Notch dynamics and SOP patterning. (A) Notch signaling couples proneural activity in neighboring cells. Cells with high proneural activity (red; Ac and Sc synergize with Sens) inhibit proneural activity in their neighbors (green) via Dl-Notch signaling and its downstream target, the E(spl) repressor. (B to G) Dynamics of Notch activity (GFP-m3) and proneural expression (Ac, Sc, Sens; Sens also labels row 5 macrochaetae) as proneural stripes emerge and resolve into SOPs (labeled by Sens). (H) Dl cis-inhibits Notch in stripe 5 (arrowhead): Notch (GFP-m3) is activated in $D l$ mutant cells (RFP-negative) along the clone border (inset). Asterisks indicate background signals. Numbering refers to the location of the proneural stripes. Scale bar, $50 \mu \mathrm{m}$. See table S1 for a full list of fly genotypes.

Fig. 2. A mathematical model. (A) Cells exhibit a bistable response to intermediate signal levels. Top: 1D landscapes depicting the dynamics of a cell for $s=0,1 / 2$, and 1 (Eqs. 2 and 3). Bottom: steady states as a function of $s$. Solid lines: stable states (valleys). Dashed line: unstable states (hill). (B) Bistability depends on the nonlinear response function $f$, which goes from 0 to 1 with $f(0)=1 / 2$ and $f^{\prime}(0)=2$. (C and $\left.\mathbf{D}\right)$ Signaling from a cell increases nonlinearly with cell state $(\mathrm{C})$ and reaches beyond immediate neighbors (D; green: signal produced by the magenta cell with $u=1)$. (E) With a receding gradient of inhibition at the boundaries, the model recapitulates patterning of rows 2-4 (green: signal; red/magenta: cell state).

Fig. 3. Attrition of the proneural group. Sens expression patterns in fixed samples (A) and live imaging of GFP-Sc (green, Histone2A-RFP, red; B) show a gradual refinement of proneural stripes. Sens expression in stripes 2 and 4 , emerging around $8 \mathrm{~h}$ APF, is sparser at $10 \mathrm{~h}$ but not fully resolved. Sc expression, which is faintly detected in a broad stripe 3 at $t_{0}+0.8 \mathrm{~h}\left(t_{0}=5-6 \mathrm{~h}\right.$ $\mathrm{APF}$ ), is restricted to a subset of cells at later stages, yet adjacent $\mathrm{Sc}$-expressing cells remain at $t_{0}+3.6 \mathrm{~h}$.

Fig. 4. Cell-intrinsic bistability. (A and $\mathbf{B}$ ) Intermediates in pattern formation, idealized as groups of equivalent cells (A, $\mathrm{A}^{\prime}$, and $\mathrm{A}^{\prime \prime}$ ), tend to steady states (colors indicate $u$ levels as in Fig. 2 ) that lie on the unstable branch of the bifurcation diagram (B; green: signaling activity; see text). (C) In a schematic time course of SOP selection (dashed line), cells are excluded from the proneural group at each step, allowing further progression of cells in the group (divergent arrows). (D) Actual cell trajectories from the model (color-coded by position within stripe 3; 
inset) show a continuous branching off of excluded cells (trajectories reverting to low $u$ ) until SOPs remain. Cells on the sides (blue), receiving a higher initial signal, are biased for early exclusion.

Fig. 5. Time courses of cell state. (A and B) The gradual resolution of stripes in the model (A) is reflected in time courses of $u$ as cells progress to different levels before they revert to low $u$ (B; a subset of stripe 3 cells represented). (C and D) Time courses of Sc expression in vivo show the same structure (C; GFP-Sc levels were quantified in a subset of stripe 3 cells from D). Colors in (A) and (C) highlight different outcomes: SOP (red/magenta); early exclusion (green); late exclusion (blue; arrowheads in B and D).

Fig. 6. Perturbation studies. Top row: model predictions. Bottom row: experiments. (A) Cells lacking Notch become SOPs (magenta) and displace neighboring stripes $(t=2)$. (A') Wild-type cells (RFP-positive) around a Notch mutant clone (dashed outline) do not express Ac (imaged live), creating a gap in stripe 2. (B) With signal levels reduced by a half, stripe 3 fails to narrow to a central row ( $t=2$; compare with Fig. $2 \mathrm{E}$ ). ( $\left.\mathbf{B}^{\prime}\right)$ In $D l$ heterozygous pupae, a disordered pattern of Ac positive and negative cells is observed in the region of stripe 3 at $8 \mathrm{~h}$ APF (compare with Fig. 1F). (C) With a reduced signaling range, stripes 2 and 4 split as lateral cells progress to high $u$ and central cells are inhibited $(t=2.5)$. $\left(\mathbf{C}^{\prime}\right)$ Live imaging of GFP-Ac in a scabrous mutant shows a splitting proneural stripe (arrowheads). (D) Without enhancement of Dl activity in SOPs, continuous bands of SOPs are formed. (D') Denser SOP rows (labeled by Sens) are observed inside a large neur mutant clone (RFP-negative cells; dashed outline). 

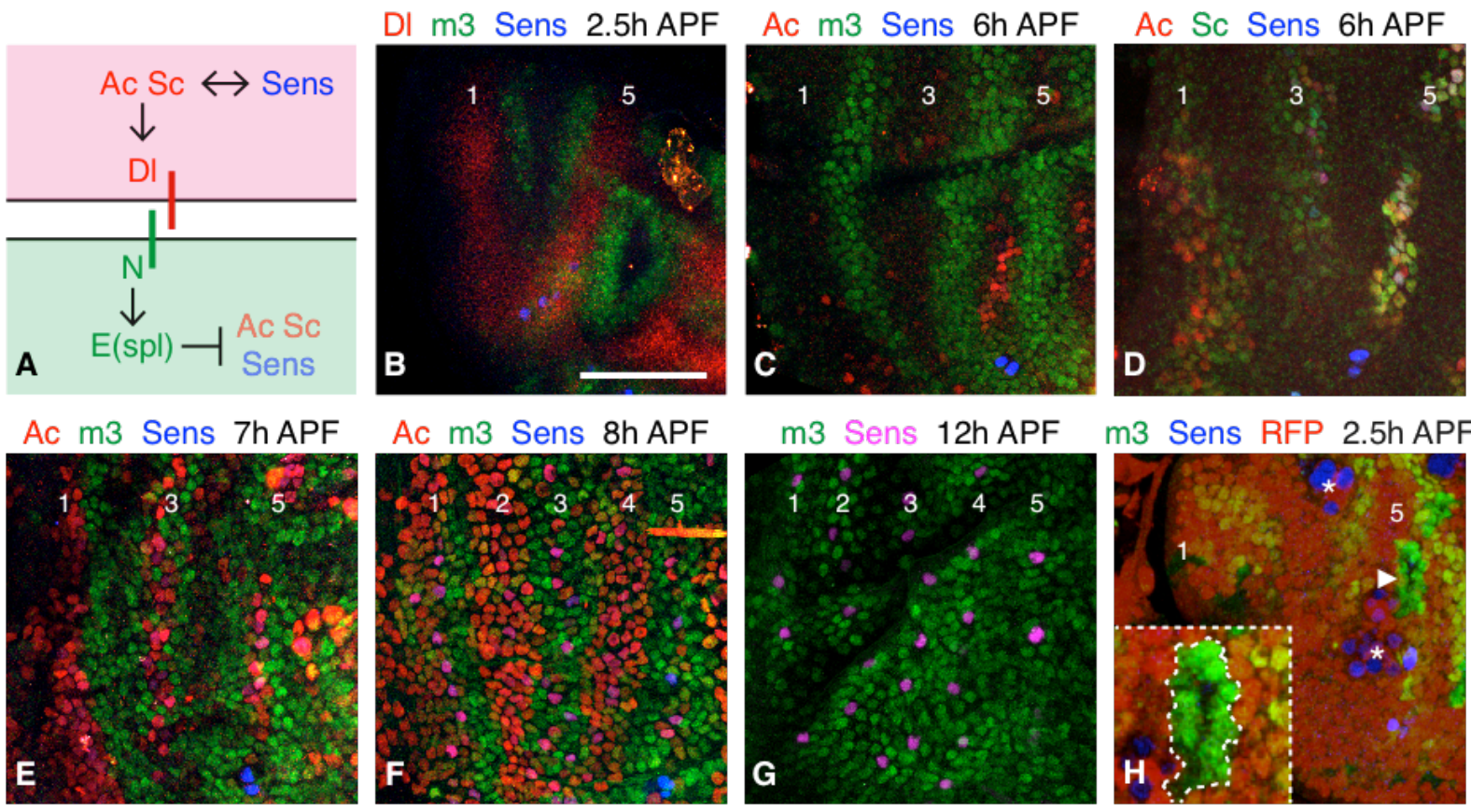

Figure 1

A

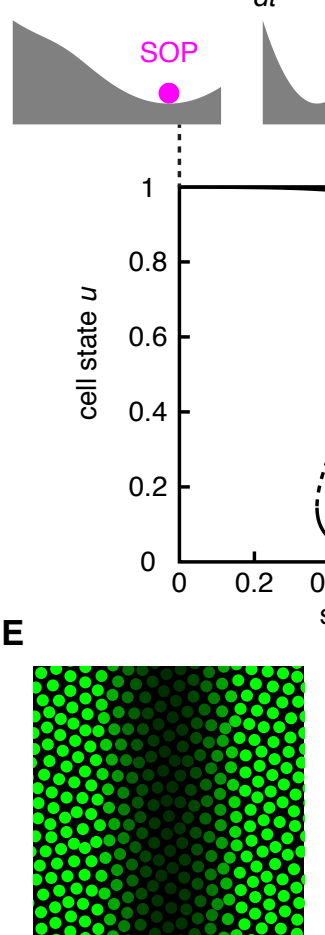

$t=0$ $\tau \frac{d u}{d t}=f(u-s)-u+\eta(t)$

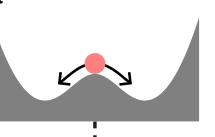

epi

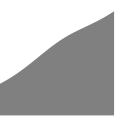

B

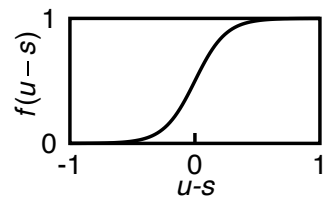

D
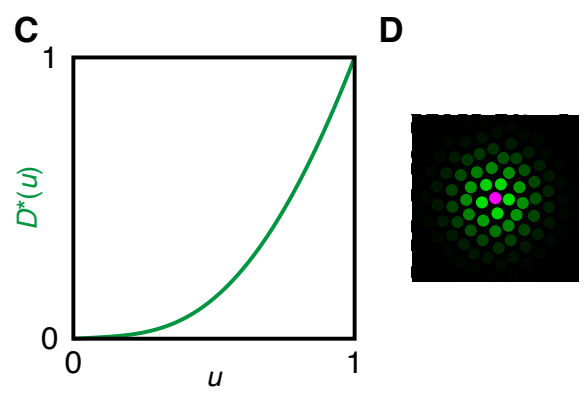

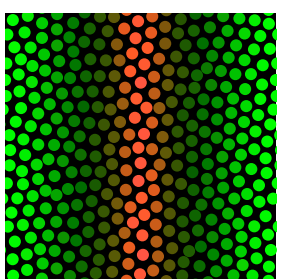

$t=1$

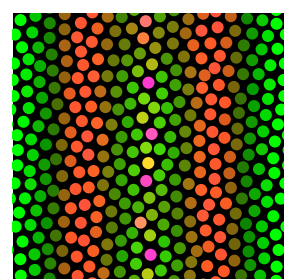

$t=2$

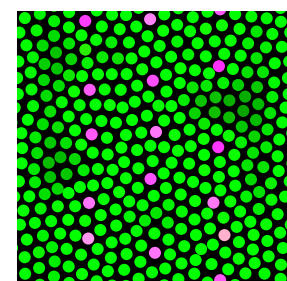

$t=5$

Figure 2 


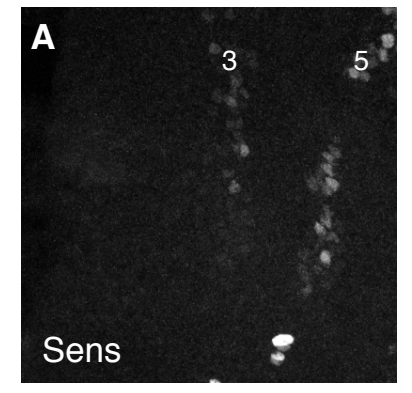

6h APF

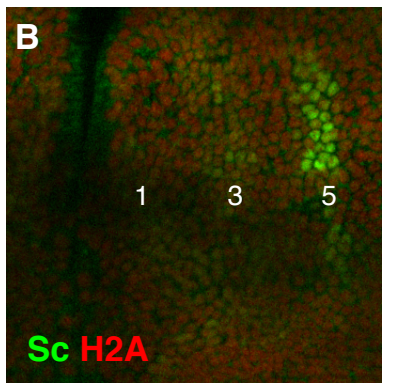

$t=t_{0}+0.8 \mathrm{~h}$

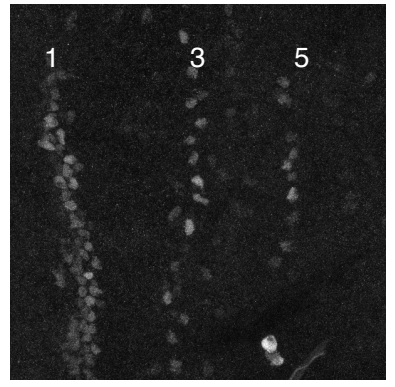

8h APF

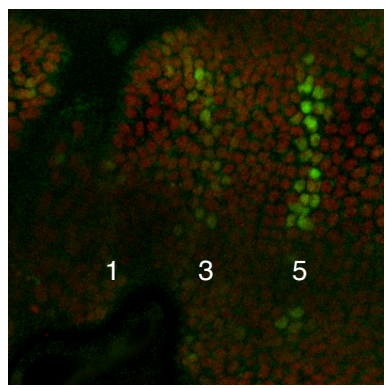

$t=t_{0}+1.7 \mathrm{~h}$

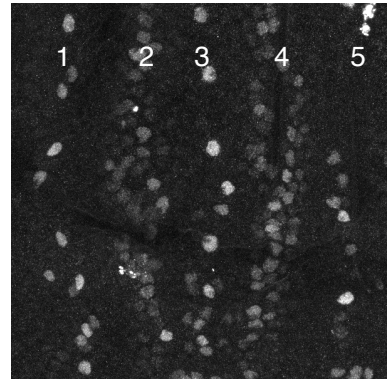

8h APF

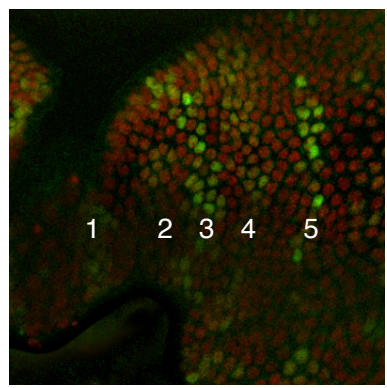

$t=t_{0}+2.7 \mathrm{~h}$

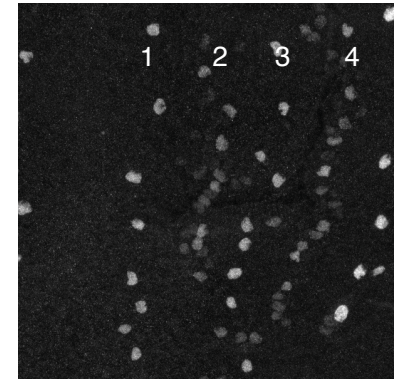

10h APF

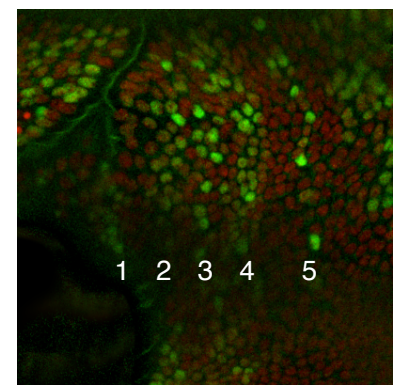

$t=t_{0}+3.6 \mathrm{~h}$

Figure 3
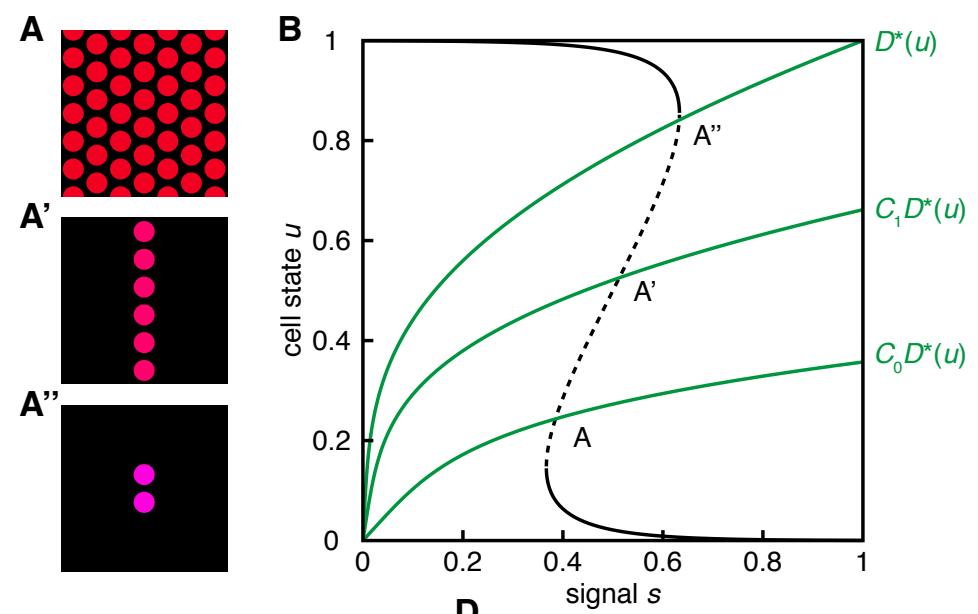

C

D

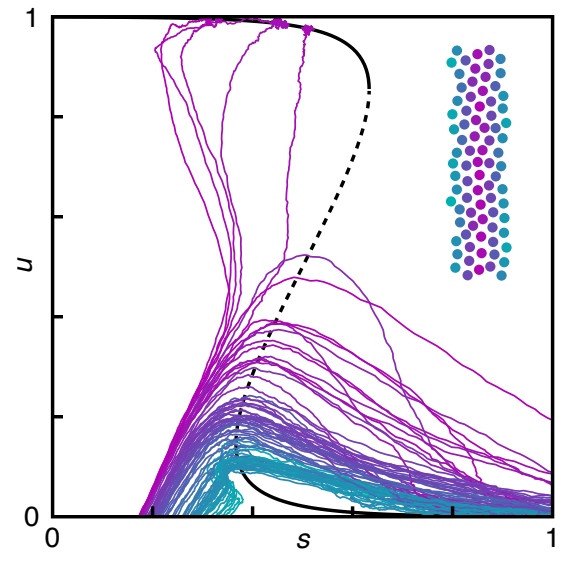

Figure 4 
A

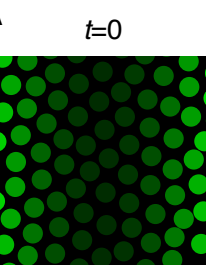

B

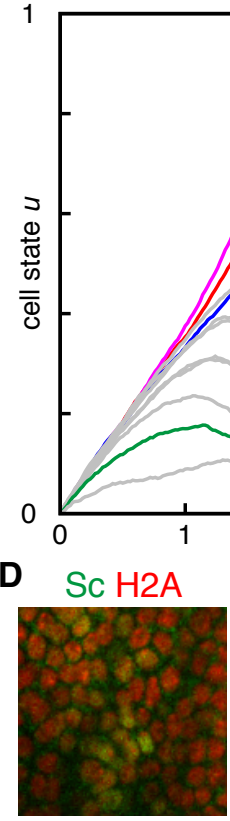

$t=t_{0}+0.6 \mathrm{~h}$ $t=1$

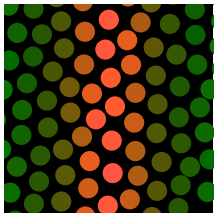

then
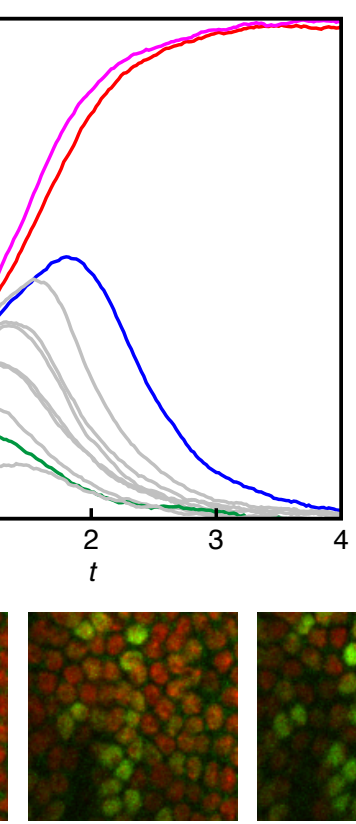

$t=t_{0}+1.4 \mathrm{~h}$ $t=1.5$

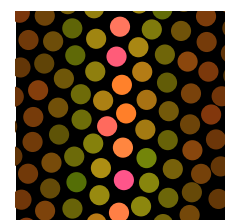

C

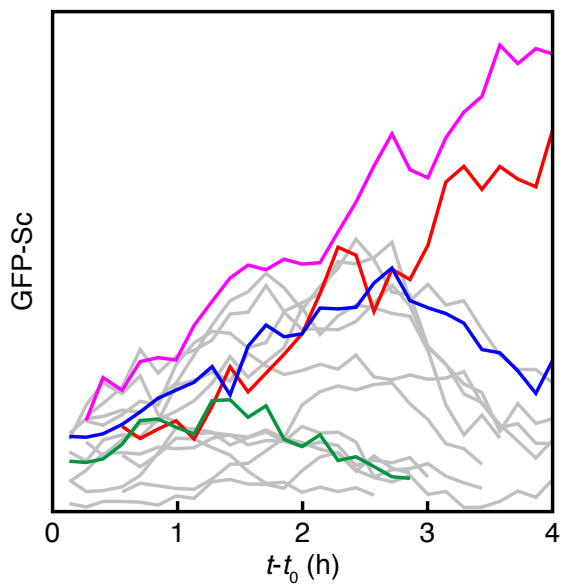

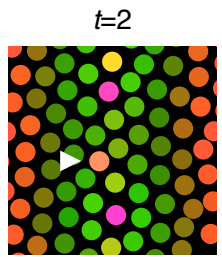
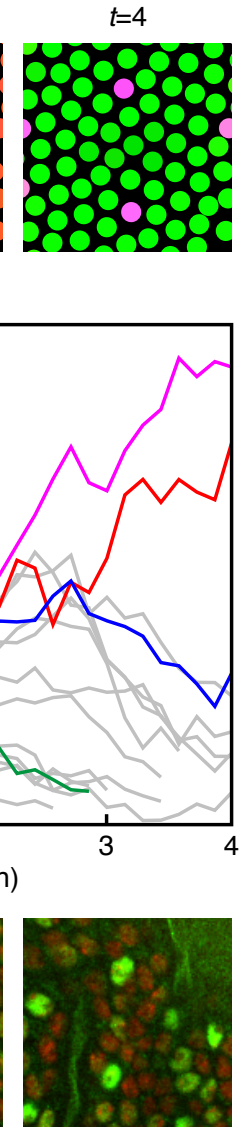

$t=t_{0}+3.1 \mathrm{~h}$

$t=t_{0}+4 \mathrm{~h}$

Figure 5

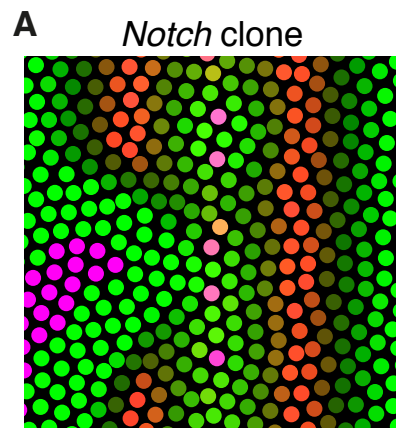

A'

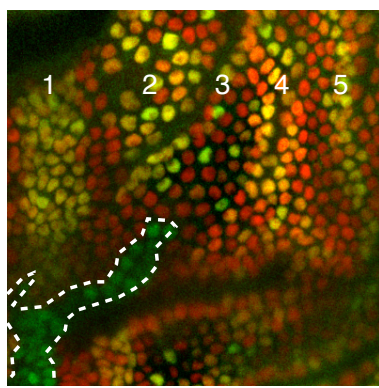

Ac RFP

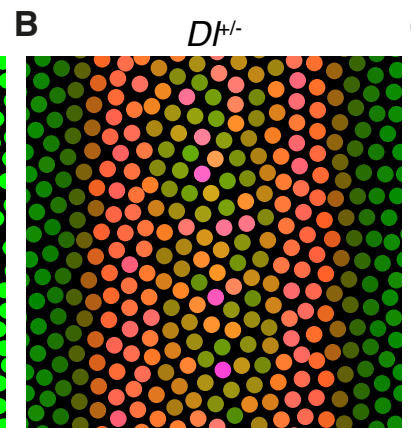

B'

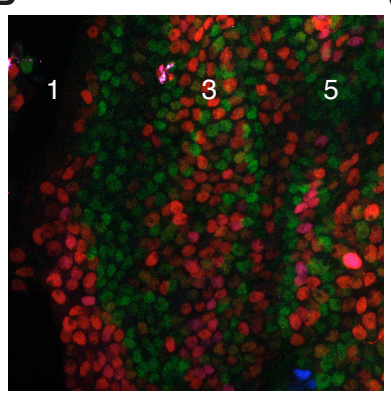

Ac m3 Sens
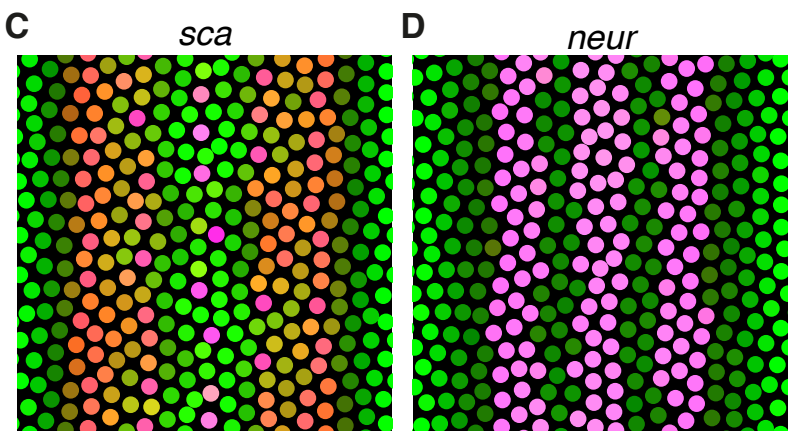

c'

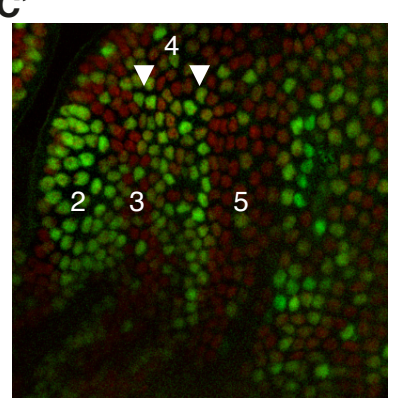

Ac $\mathrm{H} 2 \mathrm{~A}$
D

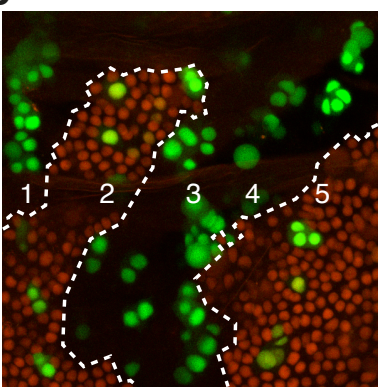

Sens RFP

Figure 6 


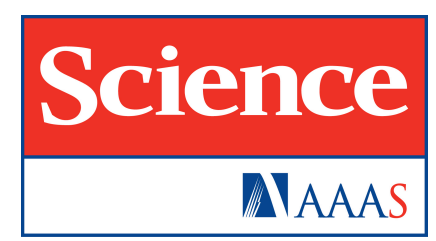

\section{Supplementary Materials for}

Self-organized Notch dynamics generate stereotyped sensory organs patterns in Drosophila

Francis Corson, Lydie Couturier, Hervé Rouault, Khalil Mazouni, François Schweisguth correspondence to: corson@lps.ens.fr; fschweis@pasteur.fr

\section{This PDF file includes:}

Supplementary Text

Figs. S1 to S13

Tables S1 and S2

Captions for Movies S1 to S5

Other Supplementary Materials for this manuscript includes the following:

Movies S1 to S5 


\section{Supplementary Text}

S1 Model with cis-inhibition

Cis-inhibition may be implicit in the bistable response of our main model

$$
\tau \frac{d u_{i}}{d t}=f\left(u_{i}, s_{i}\right)-u_{i} \equiv f\left(u_{i}, N\left(u_{i}\right) s_{i}\right)-u_{i}
$$

where the level of Notch receptor in a cell decreases as it progresses towards the SOP fate and produces more Dl. However, this does not allow for cis-inhibition of Notch independent of proneural activity, as in stripes 1 and 5 . To describe the formation of the full pattern, we extended our model with an explicit variable for Notch level.

Following $(12,40)$, we considered the dynamics of $\mathrm{Dl}$ and Notch levels within a cell as a function of $\mathrm{Dl}$ and Notch levels in neighboring cells

$$
\begin{aligned}
& \tau_{s} \frac{d N_{i}}{d t}=\beta_{N}-\gamma_{N} N_{i}-k_{c} D_{i} N_{i}-k_{t} \bar{D}_{i} N_{i} \\
& \tau_{s} \frac{d D_{i}}{d t}=\beta_{D, i}-\gamma_{D} D_{i}-k_{c} D_{i} N_{i}-k_{t} D_{i} \bar{N}_{i}
\end{aligned}
$$

where trans levels are defined through the same coupling matrix as in our main model (Eq. 9)

$$
\begin{aligned}
& \bar{N}_{i}=\sum_{j} c_{i j} N_{j} \\
& \bar{D}_{i}=\sum_{j} c_{i j} D_{j}
\end{aligned}
$$

Relative to a characteristic timescale $\tau_{s}$, Notch and Dl are produced at rates $\beta_{N}$ and $\beta_{D, i}$ and degraded at rates $\gamma_{D}$ and $\gamma_{N}$; the rate of Dl production varies between cells, while Notch production is uniform. Binding of Notch and $\mathrm{Dl}$ in cis and trans, occurring at rates $k_{c}$ and $k_{t}$, is irreversible and results in the elimination of both proteins. The signaling response of a cell is measured by the rate of trans binding, $k_{t} \bar{D}_{i} N_{i}$. With reference to Eq. S1, we define the signal $s_{i}$ as

$$
s_{i}=k_{t} \bar{D}_{i}
$$

As implicit in our main model, we take signaling to be fast relative to fate specification $\left(\tau_{s} \ll \tau\right)$, so that Notch and Dl levels are at steady state. Neglecting elimination of Notch and Dl through trans-binding, the levels of Notch and Dl in a cell depend only on Dl production in that cell:

$$
\begin{aligned}
& N_{i} \equiv N\left(\beta_{D, i}\right)=\frac{\beta_{N}-\beta_{D, i}}{2 \gamma_{N}}-\frac{\gamma_{D}}{2 k_{c}}+\sqrt{\left(\frac{\beta_{N}-\beta_{D, i}}{2 \gamma_{N}}-\frac{\gamma_{D}}{2 k_{c}}\right)^{2}+\frac{\gamma_{D} \beta_{N}}{\gamma_{N} k_{c}}} \\
& D_{i} \equiv D\left(\beta_{D, i}\right)=\frac{\beta_{D, i}-\beta_{N}}{2 \gamma_{D}}-\frac{\gamma_{N}}{2 k_{c}}+\sqrt{\left(\frac{\beta_{D, i}-\beta_{N}}{2 \gamma_{D}}-\frac{\gamma_{N}}{2 k_{c}}\right)^{2}+\frac{\gamma_{N} \beta_{D, i}}{\gamma_{D} k_{c}}}
\end{aligned}
$$

Letting Dl production depend on cell state,

$$
\beta_{D, i} \equiv \beta_{D}\left(u_{i}\right)
$$

we recovered a model of the form of Eq. S1. 
As shown in (12), with strong cis-binding cells switch from a state with high Notch and low D1 to state with low Notch and high Dl as Dl production increases (fig. S5A), and a gradient of Dl production can lead to Notch activation on the sides of a Dl stripe, similar to what we observe at early stages of patterning in the notum (Fig. 1B). However, we found that the above model could not, with the same parameter values, also account for the resolution of proneural stripes. When mutual inhibition was strong enough to exclude adjacent SOPs, Notch activity in regions with high Dl (as in stripes 1 and 5) was too strong to allow the onset of proneural activity. As with our main model, this suggested a requirement for an additional layer of regulation that enhances ligand activity as cells approach the SOP fate.

As a simplified treatment, we considered a model that combines the signal-sending curve of our main model (Eqs. 10 and 11) with varying Notch levels as above. Specifically, the level of Notch in a cell was obtained from Eq. S7 with

$$
\beta_{D, i}=\beta_{D}^{0}\left(x_{i}, t\right)+u_{i}
$$

where the time-dependent gradient $\beta_{D}^{0}(x, t)$ describes D1 expression prior to the onset of proneural activity. The signal received by the cell was defined as

$$
s_{i}=k_{t} \sum_{j} c_{i j} D_{j}^{*}
$$

where

$$
D_{i}^{*}=a\left(u_{i}\right) \beta_{D, i}
$$

and $a(u)$ is defined as in our main model, Eq. 11.

Replacing Eq. 4 with

$$
\tau \frac{d u_{i}}{d t}=\sigma\left(1.4 u_{i}-N_{i} s_{i}\right)-u_{i}+\eta_{i}(t)
$$

yielded a bifurcation diagram that is similar to our main model (fig. S5B), and we adjusted $\tau=1 / 3$ to maintain a similar pace of stripe resolution. A similar initial pattern of Notch activity (away from stripes 1 and 5) was obtained with

$$
\beta_{D}^{0}(x, t)=S_{0} \sigma\left(1-\frac{t}{\tau_{g}}\right)\left(e^{\frac{-x^{2}}{2 L^{\prime 2}}}+e^{\frac{-(1-x)^{2}}{2 L^{\prime 2}}}\right)
$$

where the range $L^{\prime}$ satisfies

$$
L^{\prime 2}+l^{2}=L^{2}
$$

and $S_{0}=2$ as in Eq. 12 (our choice of basal activity $a_{0}=.05$ yields a similar level of signal given an effective number of neighbors around 20).

This extended model recapitulated the early pattern of Notch activity in the notum (Fig. 1B,C) and the full sequence of five proneural stripes and SOP rows (fig. S5C). Thus, a transient gradient of Dl expression is sufficient to establish the pattern of microchaeta rows.

\section{$\underline{\text { S2 }}$ Monostable model}

Our main model (Eqs. 4-11) exhibits a bistable response: for certain values of the signal $s$, a cell admits two stable states, with low $u$ and high $u$ (Fig. 2A). Analysis of its patterning behavior suggests an essential role for cell-instrinsic bistability in the 
dynamics of SOP selection (Figs. 3-5). In support of this analysis, we also considered a monostable model, in which a cell admits a single stable state for every value of the signal.

Many models for lateral inhibition patterning, e.g. (36), exhibit a monostable response because they incorporate no cell-intrinsic feedback. In terms of the generic model

$$
\tau \frac{d u_{i}}{d t}=f\left(u_{i}, s_{i}\right)-u_{i}
$$

the absence of cell-intrinsic feedback can be stated as

$$
f\left(u_{i}, s_{i}\right) \equiv f\left(s_{i}\right)
$$

such that

$$
\tau \frac{d u_{i}}{d t}=f\left(s_{i}\right)-u_{i}
$$

and $u_{i}=f\left(s_{i}\right)$ at steady state.

Generically, the linear stability analysis of section S3 implies that patterning with a monostable response requires strong nonlinearities in cell-cell signaling, i.e. a sharp onset of signaling from presumptive SOP and/or a very sensitive response in the receiving cells. As an illustration, we considered a model of the form

$$
\tau \frac{d u_{i}}{d t}=\sigma\left(c\left(s_{c}-s_{i}\right)\right)-u_{i}+\eta_{i}(t)
$$

where $\sigma$ is the sigmoidal function defined by Eq. 7. With this definition, the steady state of a cell switches from high to low $u$ with a slope $c$ as the signal crosses the threshold $s_{c}$. We set a moderate slope, $c=4$, and $s_{c}=.2$, yielding an initial profile for stripe 3 that is similar to our main model.

We considered two variations of this model. In the first (fig. S7), we kept the same, relatively smooth, signal-sending curve as in our main model, Eqs. 10 and 11; the uniform steady state was stable and no pattern developed (fig. S7B). In the second (fig. S8), we considered a sigmoidal signal-sending curve

$$
D^{*}(u)=\sigma\left(k\left(u-u_{c}\right)\right)
$$

that steps from 0 to 1 with a slope $k$ at the threshold $u=u_{c}$. We set $u_{c}=.4$, such that ligand production switches on at intermediate levels of proneural activity. As implied by the linear stability analysis of section S3, a steep slope was required for patterning and we set $k=8$. Although this model yielded a pattern (fig. S8C), the dynamics of SOP selection were very different. Isolated SOPs emerged from a relatively uniform and steady background of proneural activity (fig. S8B,C), in contrast with the gradual narrowing of the proneural group in our main model and in experiments (Figs. 3 and 5).

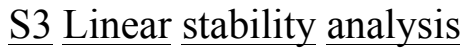

The behavior of a pattern-forming system can be analyzed in terms of the response of a uniform steady state to small perturbations (51); if some perturbations are amplified, the uniform steady state is unstable and a pattern will spontaneously emerge in the presence of fluctuations. Such a linear stability analysis has been described previously for a generic lateral inhibition model (52). Here, we specialize the analysis to a model with a single state variable and instantaneous signaling, Eqs. 4 and 8. The response to 
perturbations can be computed analytically if cells are arranged in a regular, hexagonal lattice with spacing $a$ :

$$
\vec{r}_{i}=p_{i} \vec{a}_{1}+q_{i} \vec{a}_{2}
$$

where $p_{i}$ and $q_{i}$ are two integers and $\left\|\vec{a}_{1}\right\|=\left\|\vec{a}_{2}\right\|=a$.

Let $u^{*}$ and $s^{*}$ denote the cell state and signal level in a uniform steady state, satisfying $f\left(u^{*}, s^{*}\right)=u^{*}$, and consider a small perturbation

$$
u_{i}=u^{*}+\delta u_{i}
$$

Linearizing the model equations (Eqs. 4 and 8 ) yields

$$
\tau \frac{d}{d t} \delta u_{i}=\alpha \delta u_{i}+\beta \gamma \sum_{j} c_{i j} \delta u_{j}
$$

where

$$
\begin{gathered}
\alpha=\frac{\partial f}{\partial u}-1 \\
\beta=\frac{\partial f}{\partial s}<0 \\
\gamma=\frac{d}{d u} D^{*}(u)
\end{gathered}
$$
form

An arbitrary perturbation can be decomposed into sinusoidal (plane) waves of the

$$
\delta u_{i}=\sin \left(\vec{k} \cdot \vec{r}_{i}+\phi\right)=\sin \left(k_{x} x_{i}+k_{y} y_{i}+\phi\right)
$$

Each of these modes grows at a rate

$$
\frac{\alpha+\beta \gamma \hat{c}(\vec{k})}{\tau}
$$

where $\hat{c}(\vec{k})$ is the (periodic) Fourier transform of the (discrete) coupling function $c(\vec{r})$,

$$
c(\vec{r})=\sum_{(p, q) \neq(0,0)} g\left(p \vec{a}_{1}+q \vec{a}_{2}, l^{2}\right) \delta\left(\vec{r}-p \vec{a}_{1}-q \vec{a}_{2}\right) a
$$

with

$$
g\left(\vec{r}, \sigma^{2}\right)=e^{\frac{-\vec{r}^{2}}{2 \sigma^{2}}}
$$

so that

$$
\hat{c}(\vec{k})=-1+\frac{4 \pi l^{2}}{a^{2} \sqrt{3}} \sum_{p, q} g\left(\vec{k}-p \vec{b}_{1}-q \vec{b}_{2}, \frac{1}{l^{2}}\right)
$$

In this equation, $\vec{b}_{1}$ and $\vec{b}_{2}$ are the primitive vectors of the reciprocal lattice, satisfying

$$
b \equiv\left\|\vec{b}_{1}\right\|=\left\|\vec{b}_{2}\right\|=\frac{4 \pi}{a \sqrt{3}}
$$

A similar stability analysis can be conducted for a 1D file of cells with the same spacing $a$. The growth rate of a perturbation with wavenumber $k$ is given by 


$$
\frac{\alpha+\beta \gamma \hat{c}(k)}{\tau}
$$

where $\hat{c}(k)$ denotes the Fourier transform of the relevant 1D coupling function,

$$
\hat{c}(k)=-1+\sqrt{2 \pi} \frac{l}{a} \sum_{p} e^{\frac{-p^{2} l^{2} k^{2}}{2}}
$$

As noted in previous studies of discrete pattern-forming systems $(52,53)$, the growth rate of perturbations, Eq. S28, breaks down into two contributions, corresponding to cellintrinsic dynamics and cell-cell interactions. Fig. S9A,B shows the contribution of lateral inhibition alone $(\beta \gamma<0$ thus the negative of $\hat{c}(\vec{k})$ must be considered). Small-scale perturbations grow at a rate that is limited by $\hat{c}(\vec{k})>-1$, while large-scale perturbations decay at rate that is related to the effective number of neighbors $C_{0}$ to which a cell is coupled,

$$
\hat{c}(0)=\sum_{j} c_{i j} \equiv C_{0}
$$

With a finite signaling range, $C_{0}$ can be much larger than one (with our parameter values, $C_{0} \approx 18$ ), thus large-scale perturbations are much more strongly damped than small-scale perturbations are amplified.

With a bistable response, cell-intrinsic dynamics are destabilizing $(\alpha>0)$, thus the uniform steady state is always unstable, and the range of unstable modes is increased relative to a system without cell-intrinsic feedback (fig. S9C). Although small-scale perturbations grow fastest, an external field can drive the emergence of a large-scale pattern before small-scale fluctuations are amplified.

With a monostable response, cell-intrinsic dynamics are stabilizing $(\alpha<0)$, thus the range of unstable modes is reduced (fig. S9D) and instability of the uniform steady state depends on the strength of lateral inhibition. In the case where $f(u, s) \equiv f(\mathrm{~s})$ (cf. section $\mathrm{S} 2$ ), $\alpha=-1$ and $\beta=f^{\prime}$. From Eq. S28 and $\hat{c}(\vec{k})>-1$, instability of the uniform steady state requires $|\beta \gamma|>1$, which we can rewrite as

$$
\left|\frac{f^{\prime} D^{* \prime}}{D^{*}}\right|>\frac{1}{D^{*}}=\frac{C_{0}}{s^{*}}
$$

With our choice of units, the steady-state signal $s^{*}$ is a fraction of unity, and with a finite signaling range, the effective number of neighbors $C_{0}$ is large. Thus, the above inequality implies strong nonlinearities in the signal-sending curve $D^{*}$ and/or the response curve $f$.

Although nonlinearity is essential for stripe resolution (narrowing of the proneural group is essentially a nonlinear phenomenon), this linear analysis provides a complementary justification of the requirement for bistability. A newly-formed proneural stripe can be envisioned as a moderate disturbance relative to a uniform state, on a spatial scale that is comparable with the signaling range; in a bistable model this can be autonomously maintained (and later refined; fig. S9E), while in a monostable model it is rapidly suppressed (and SOPs emerge through the amplification of small-scale fluctuations; fig. S9F). 
A

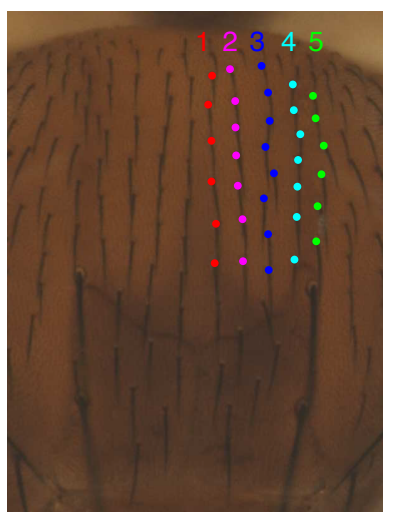

B

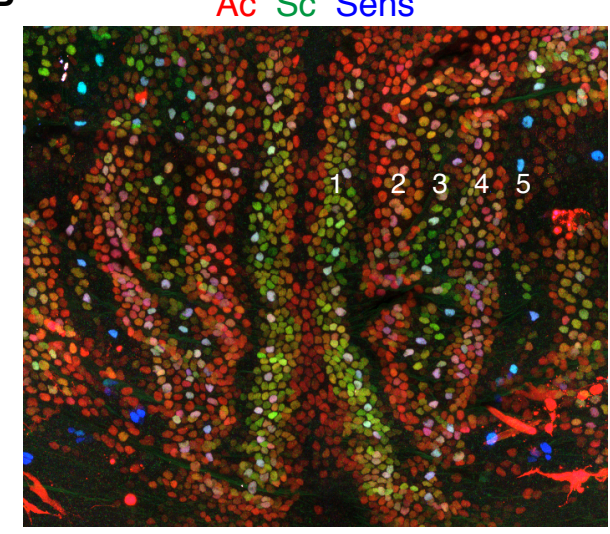

C
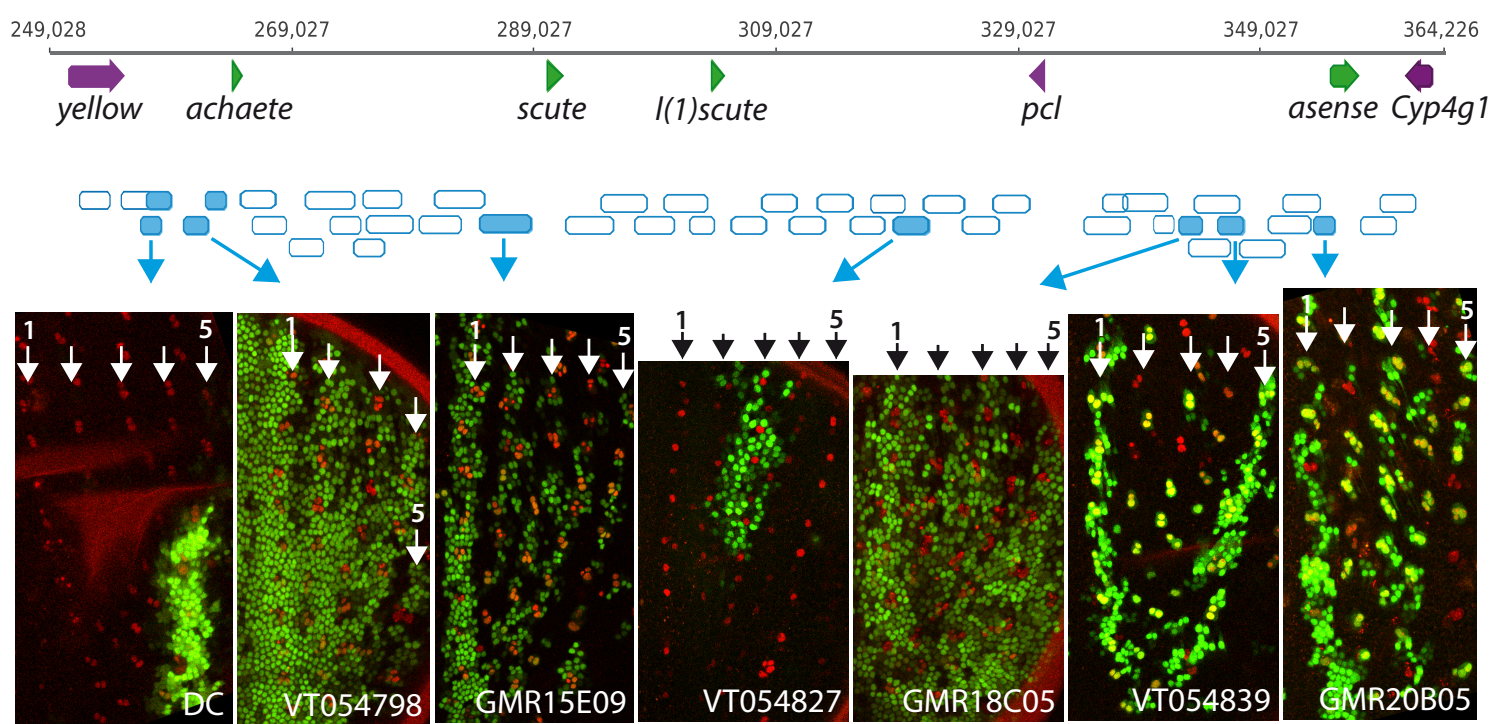

Fig. S1. Bristle rows and proneural stripes: analysis of the $A S$ - $C$ fails to identify stripe-specific enhancers.

$(\mathbf{A}, \mathbf{B})$ The fly notum exhibits five rows of bristles on each side of the dorsal-central region (A). These develop from five proneural stripes expressing Ac and Sc (B; emerging SOPs are marked by Sens). Stripes and rows are numbered from 1 to 5 along the medialdistal axis, as indicated (A,B). (C) The cis-regulatory activity of 46 fragments covering the whole $A S-C$ (corresponding to publicly available enhancer-Gal4 lines) was determined in early pupae (using a UAS-nuclearGFP reporter line). The position of these fragments (blue boxes) is shown onto the genomic map of the $A S-C$. This analysis detected seven enhancers but failed to identify enhancers that are specifically active in individual stripes. This suggests that the pattern of five proneural stripes seen in the notum is unlikely to result from the composite addition of individual stripes, each stripe being defined based on its position along the medial-distal axis. 
A

B

C

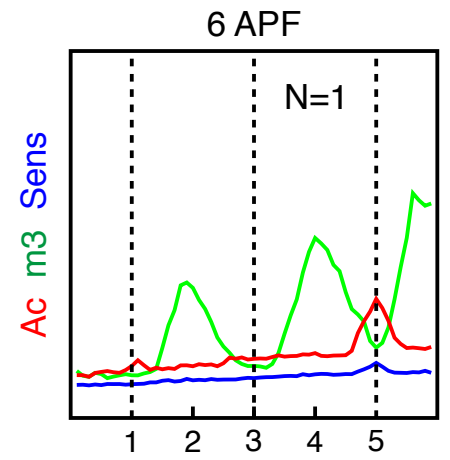

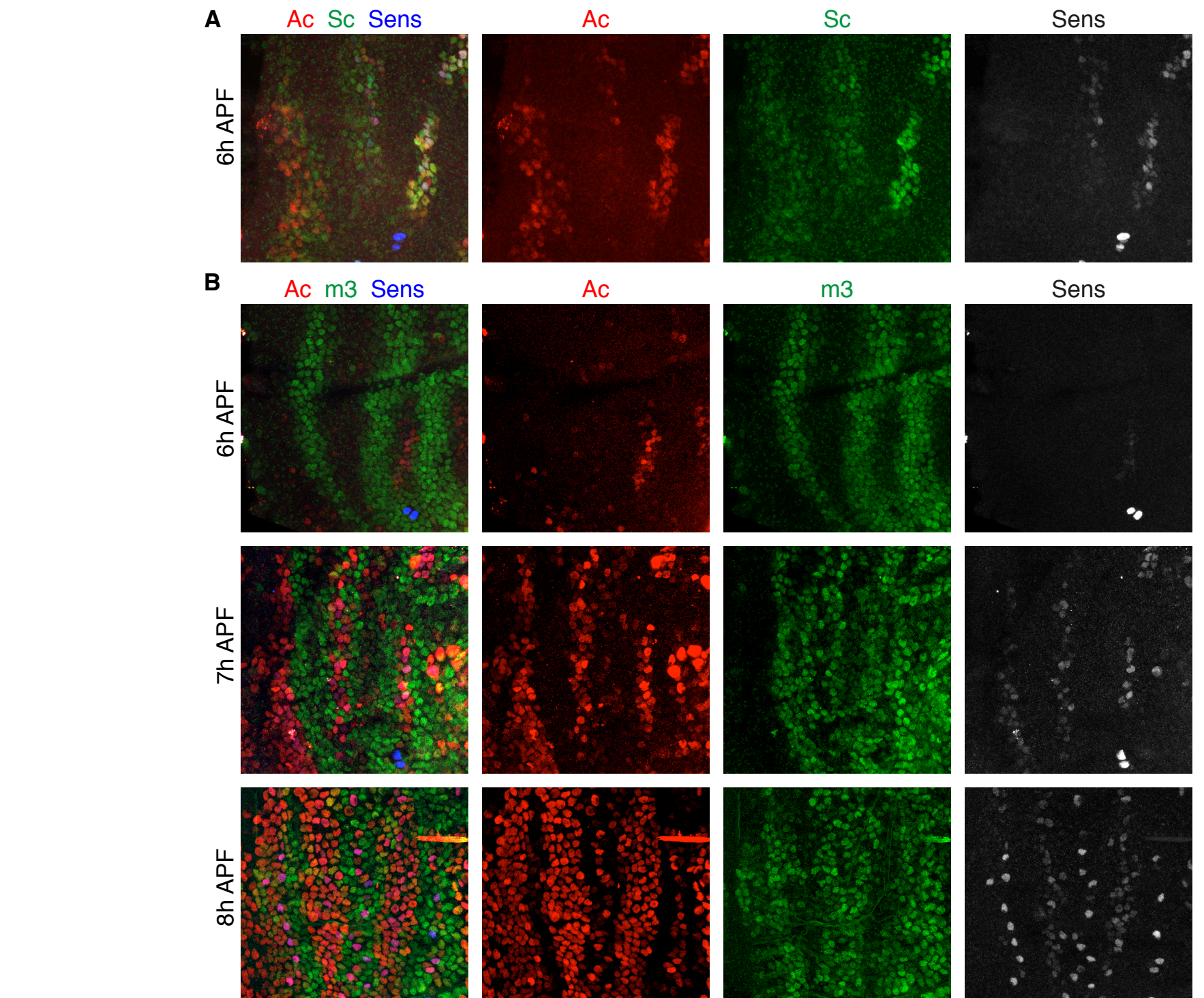
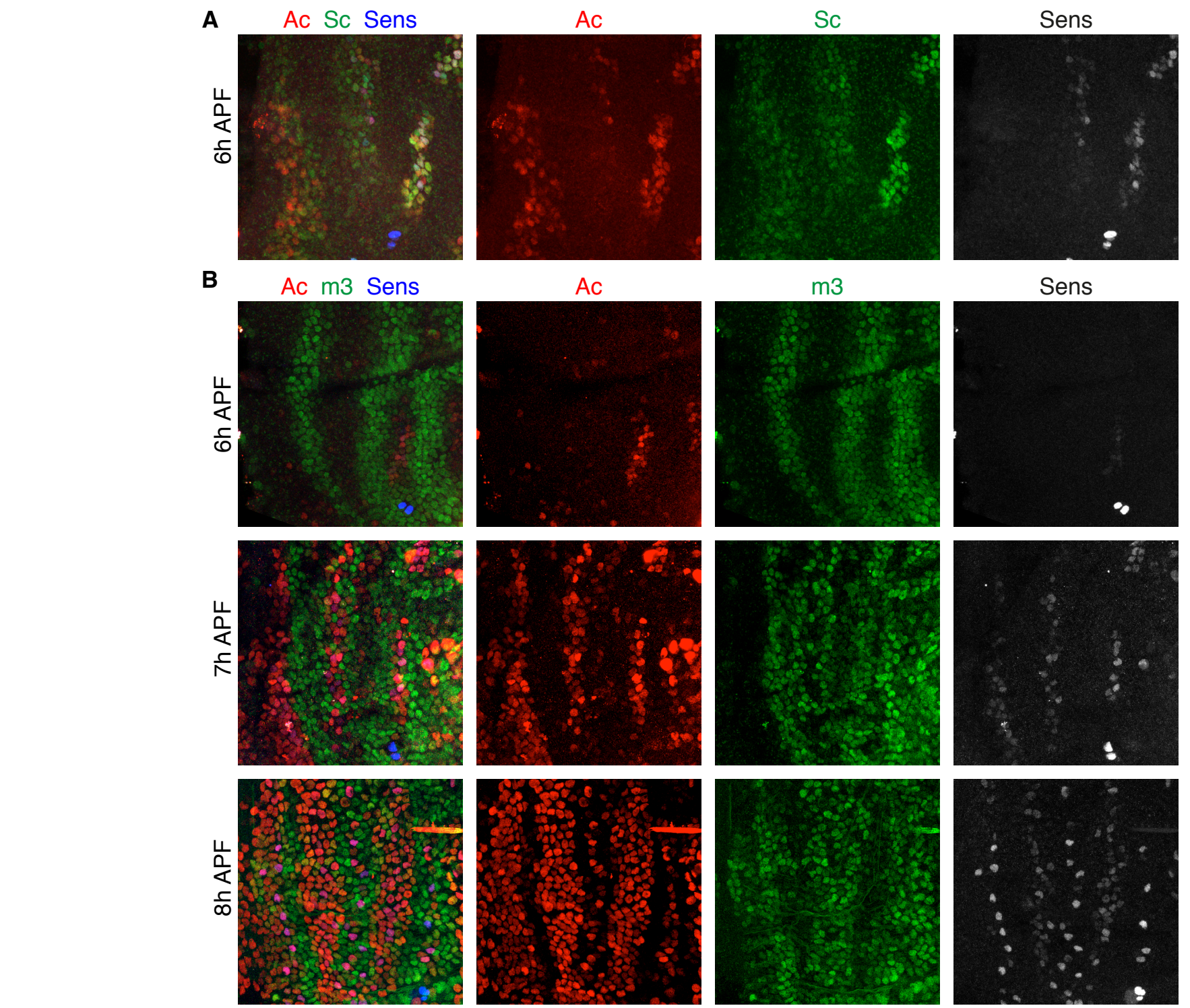

Ac

Ac

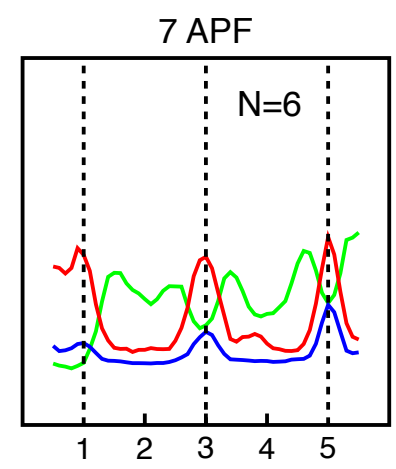

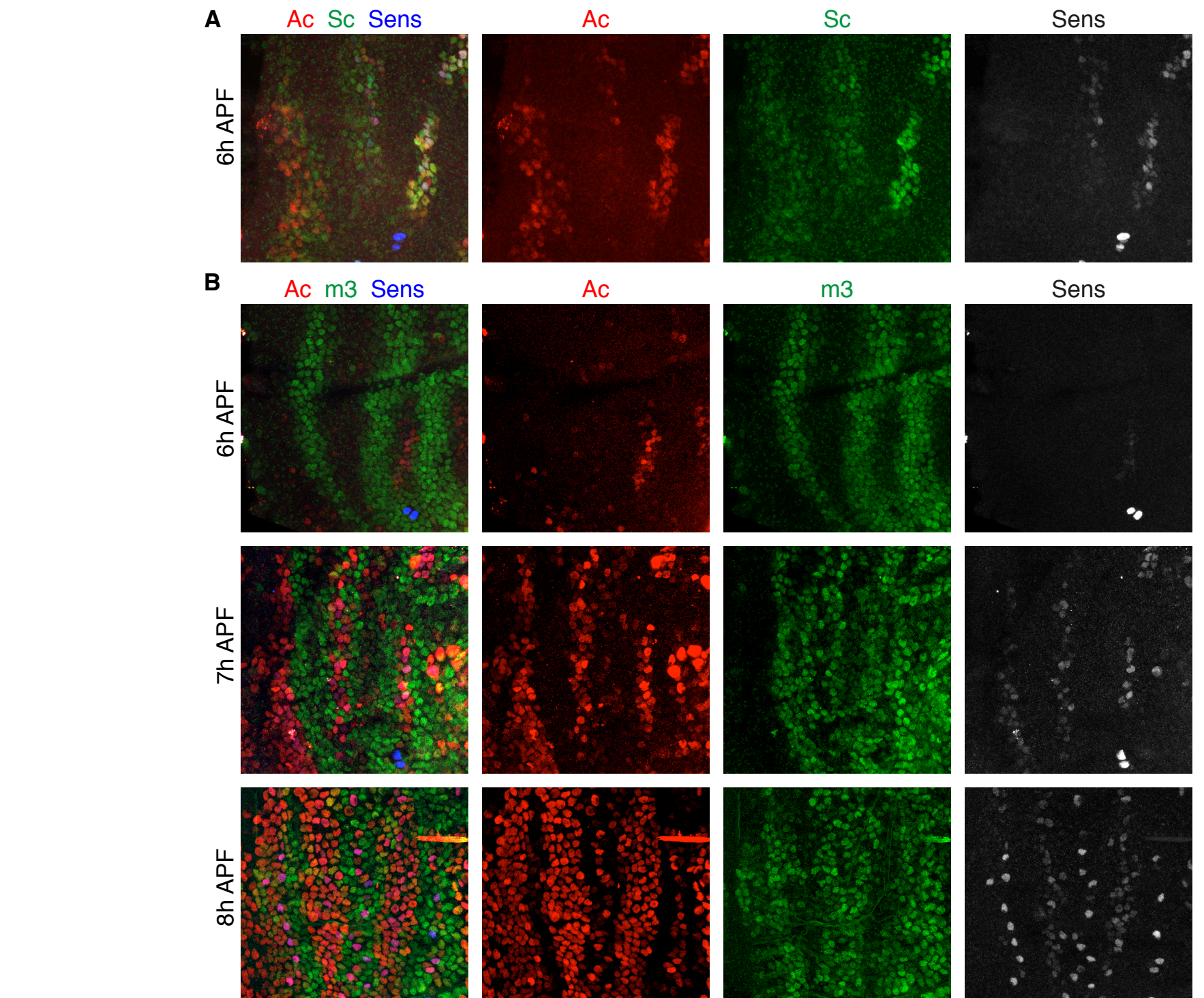
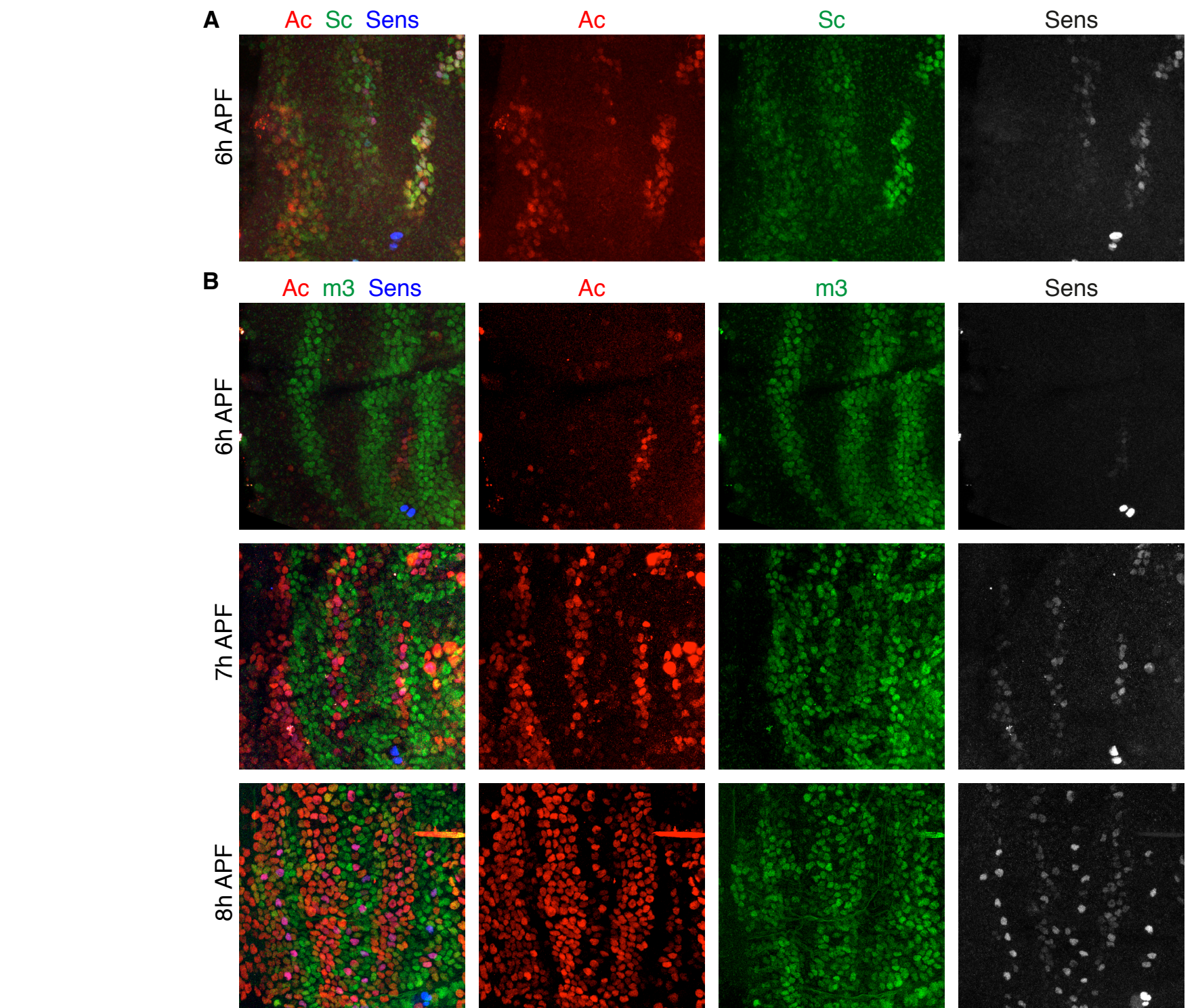

Sc

m3

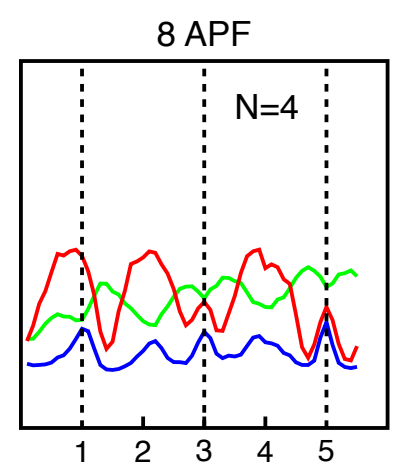

Sens

Sens
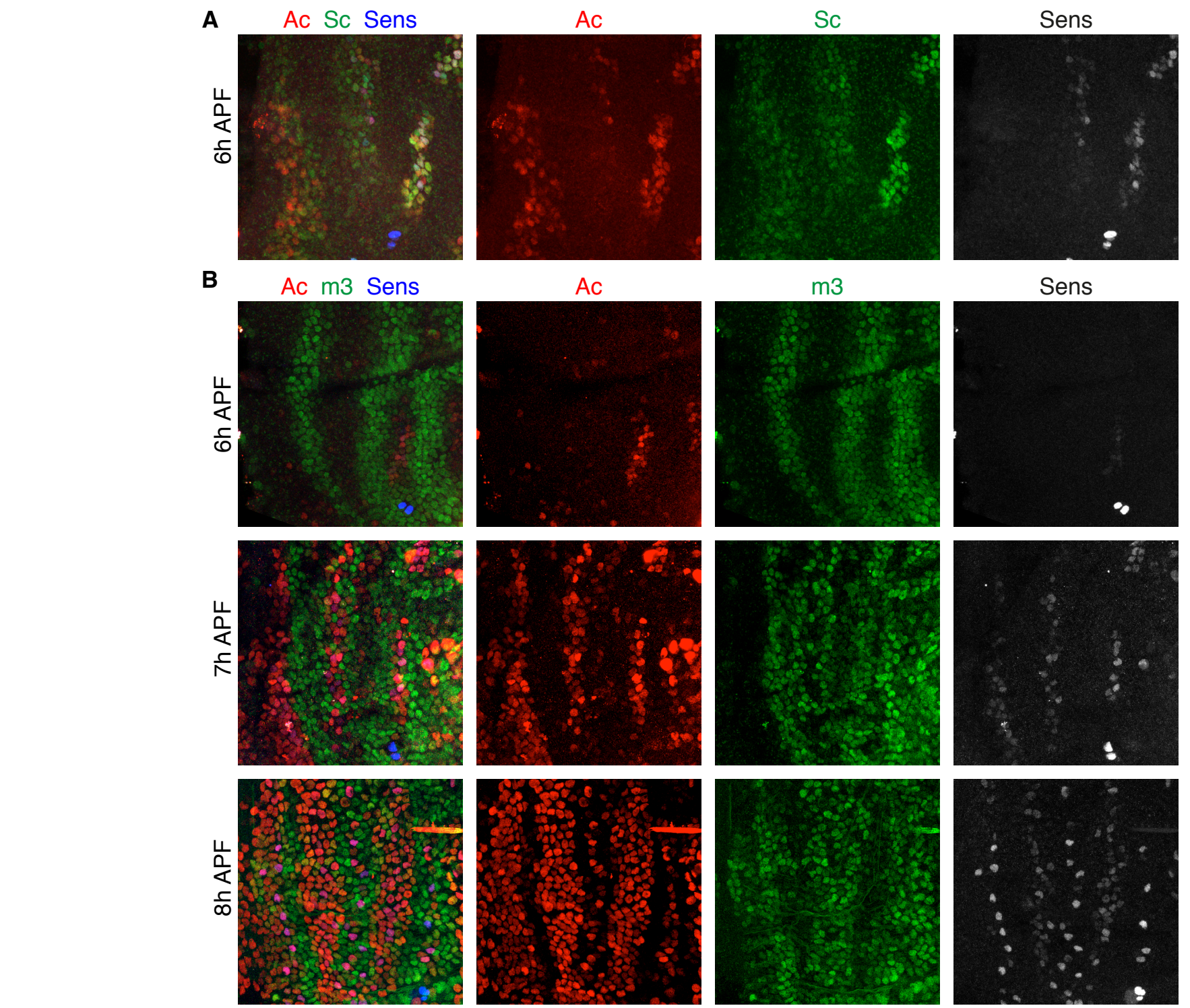
(B) Expression patterns of Ac (Cherry-Ac, red), E(spl)m3-HLH (GFP-m3, green) and Sens (blue) at successive time points (cf. Fig. 1C,E,F). (C) Profiles of Notch (GFP-m3) and proneural activity (Ac and Sens) at successive time points. Profiles are averages over several images ( $\mathrm{N}$ images, as indicated). Images were manually annotated to specify a region of interest and the centerlines of the stripes, defining a continuous medial-distal coordinate with integer values at the centers of the stripes. Profiles along this coordinate were computed from maximum projections of the images. 

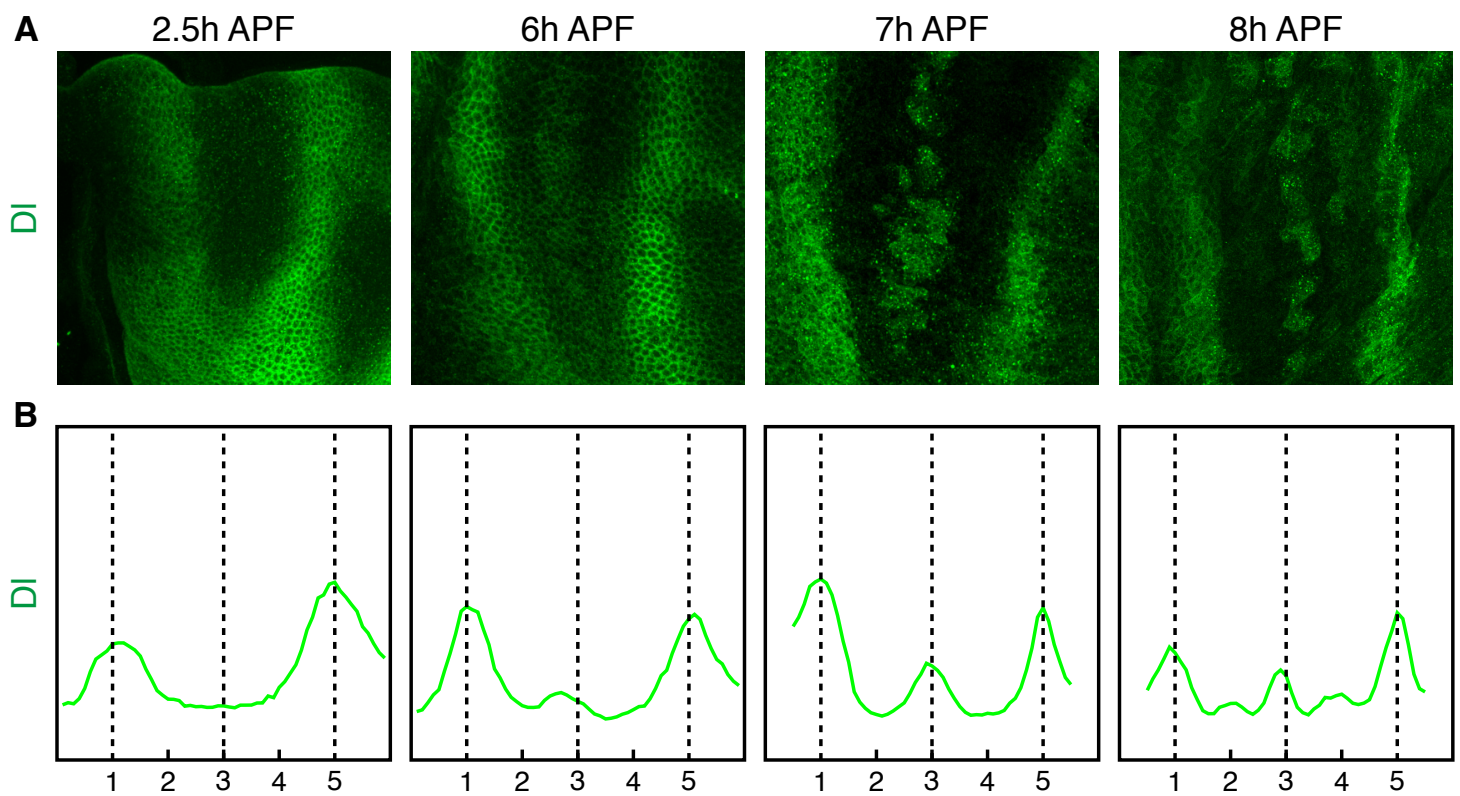

Fig. S3. Dynamics of DI expression in the notum.

(A) Expression patterns of Dl at successive time points (cf. Fig. 1B,C). At 2.5 h APF, D1 exhibits a smoothly varying pattern, with peak levels at the locations of the future stripes 1 and 5. At $6 \mathrm{~h} \mathrm{APF}$, a stripe of Dl expression appears in the center of the dorso-central region. At $7 \mathrm{~h} \mathrm{APF}$, the initially smooth pattern of $\mathrm{Dl}$ is replaced by a more discontinuous pattern. This pattern further evolves with the emergence of two faint intercalating stripes of Dl at $8 \mathrm{~h} \mathrm{APF}$. (B) Corresponding profiles of Dl expression, determined from the images in (A) as described in the caption of fig. S2C. 

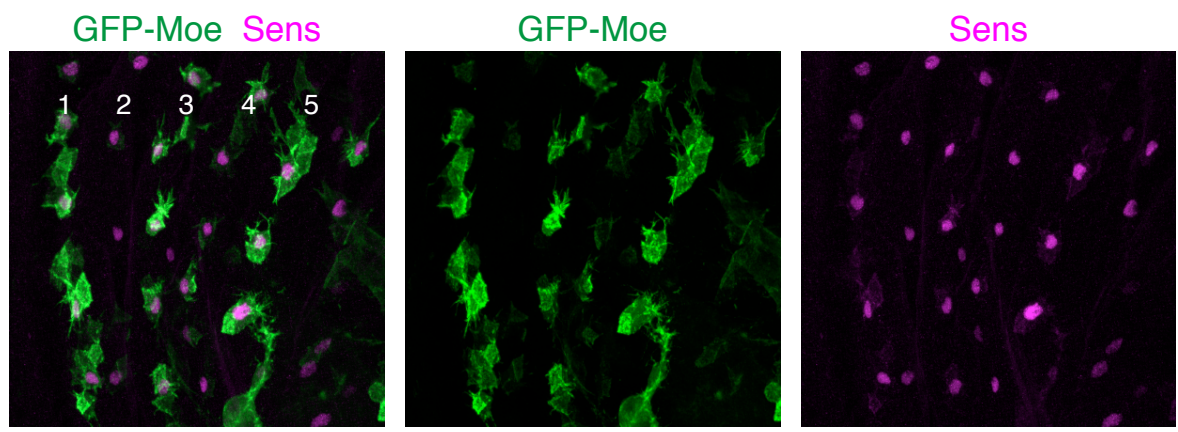

Fig. S4. Comparison of Sens and GFP-Moe patterns suggests limited pattern refinement after $12 \mathrm{~h}$ APF.

Expression patterns of Sens and neur $>$ GFP-Moe at $12 \mathrm{~h}$ APF. GFP-Moe is expressed more broadly than Sens at $12 \mathrm{~h}$ APF. Cells expressing Sens are regularly spaced. By contrast, the GFP-Moe pattern is not fully resolved: in stripes 2 and 4, Sens-positive SOPs start to express GFP-Moe whereas Sens-negative cells located in stripes 1, 3, and 5 still express GFP-Moe. Thus, refinement of the pattern after $12 \mathrm{~h}$ APF may be much more limited than inferred from GFP-Moe expression (11). 
A

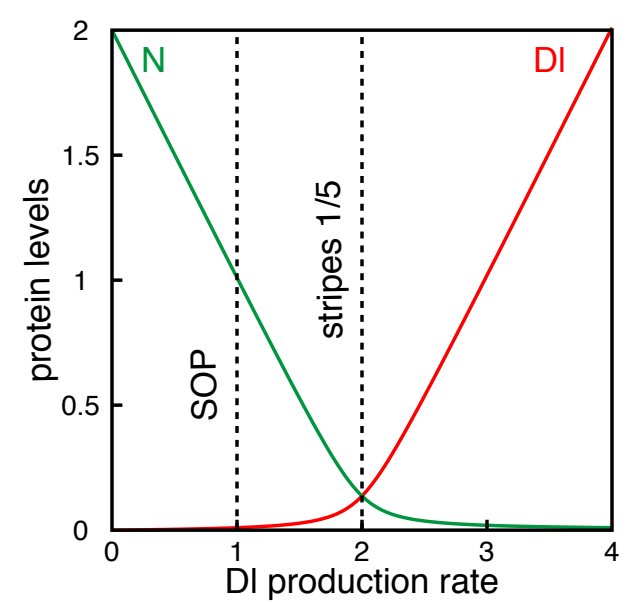

C

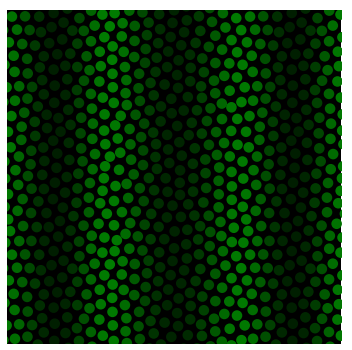

$t=0$

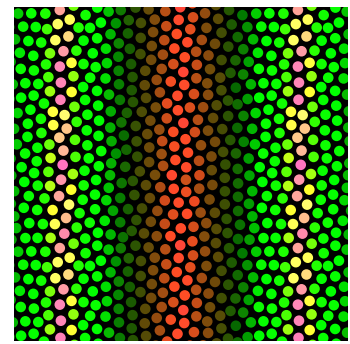

$t=1$
B
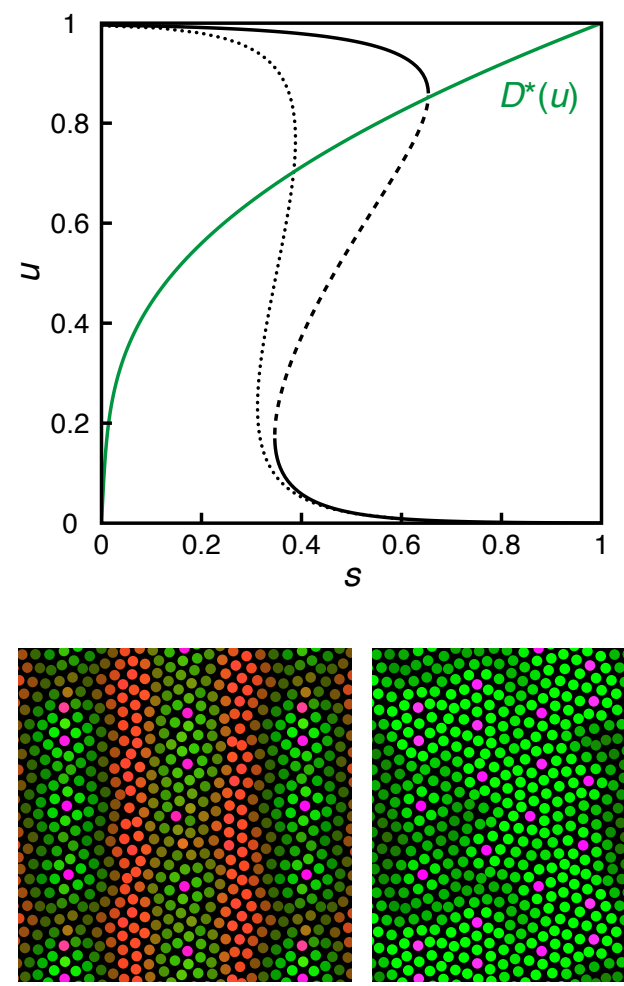

$t=2$

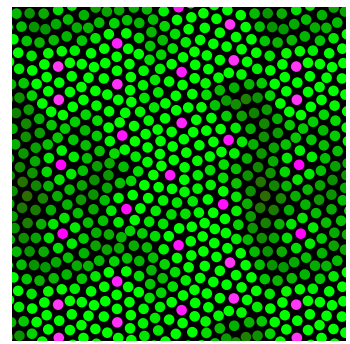

$t=5$

Fig. S5. Incorporation of cis-inhibition in the model.

(A) With cis-inhibition and assuming a constant rate of Notch production, the level of Notch receptor in a cell decreases with its rate of Dl production (12). (B) Bifurcation diagram of our extended model incorporating cis-inhibition. The model (see supplementary text S1) integrates varying Notch receptor levels, as in (A), with an unchanged signal-sending curve (green). The dotted line shows the steady states in the absence of cis-inhibition (i.e. $N_{i}=\beta_{N} / \gamma_{N}=2$ in Eq. S13), with a bistable response in a narrow range of signal levels. With cis-inhibition (extended model, corresponding to the dashed and solid lines), a higher level of signal (trans Dl) is required for the same Notch activity and cell state $u$, yielding a larger bistable range, similar to our main model without cis-inhibition (compare with Fig. 2A). (C) Patterning dynamics of the extended model. If we simply specify an initial gradient of Dl as observed, the model can account for the initial pattern of Notch activity (green, $t=0$ ), with low activity in the region of stripes 1 and 5, and the sequential emergence of five proneural stripes ( $t=1$ and $t=2)$ and SOP rows $(t=5)$. Here, the initial, proneural-independent Dl production in stripes 1 and 5 (Eq. S14 with $S_{0}=2$ ) is taken to be sufficient to strongly reduce Notch levels, while D1 production in a SOP (Eq. S10 with $u \leq 1)$ is smaller and yields only a partial reduction in Notch level (see dashed lines in A). 
A Ac Sc Sens 8h APF
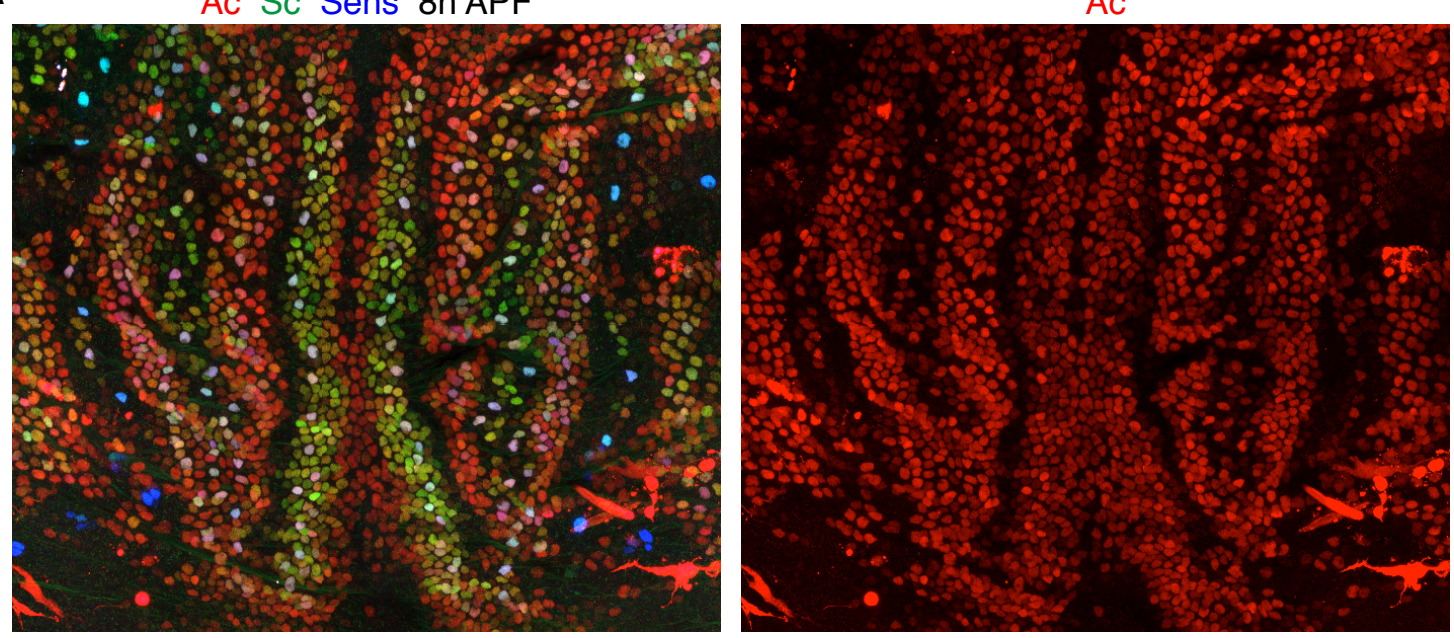

Sc
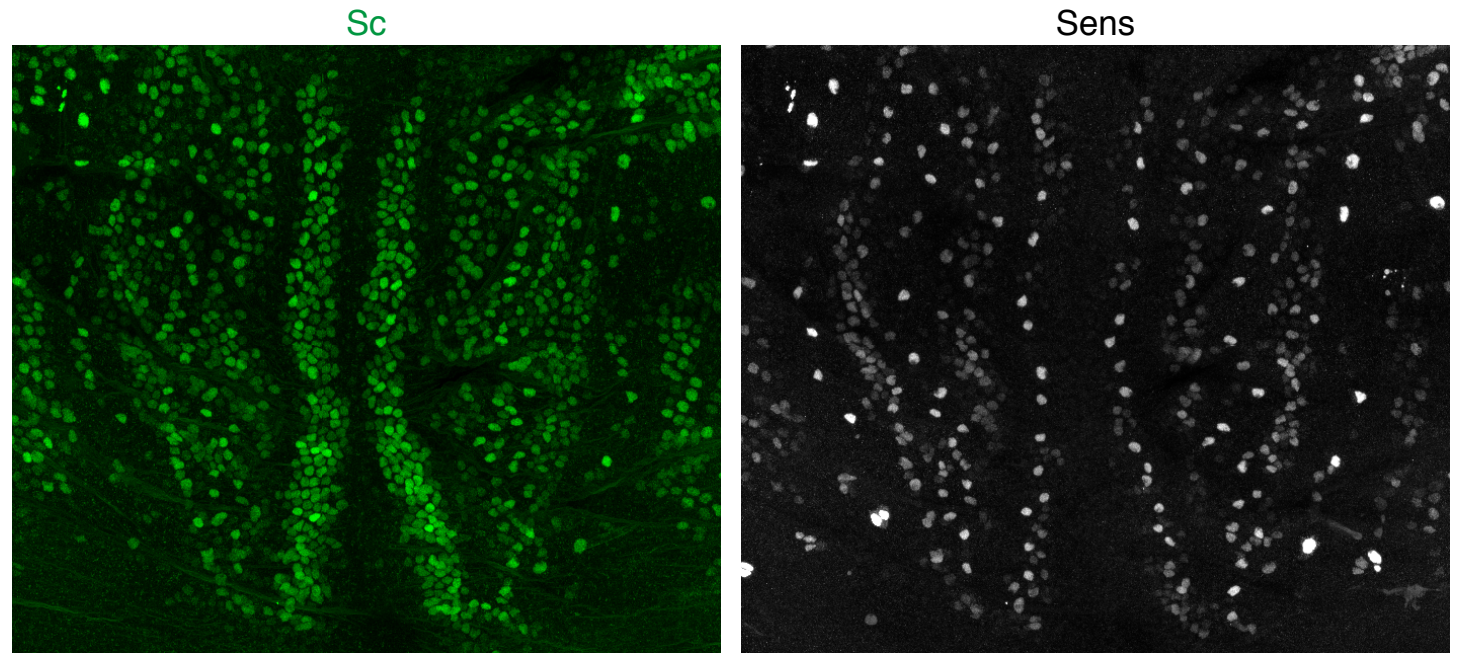

B

C
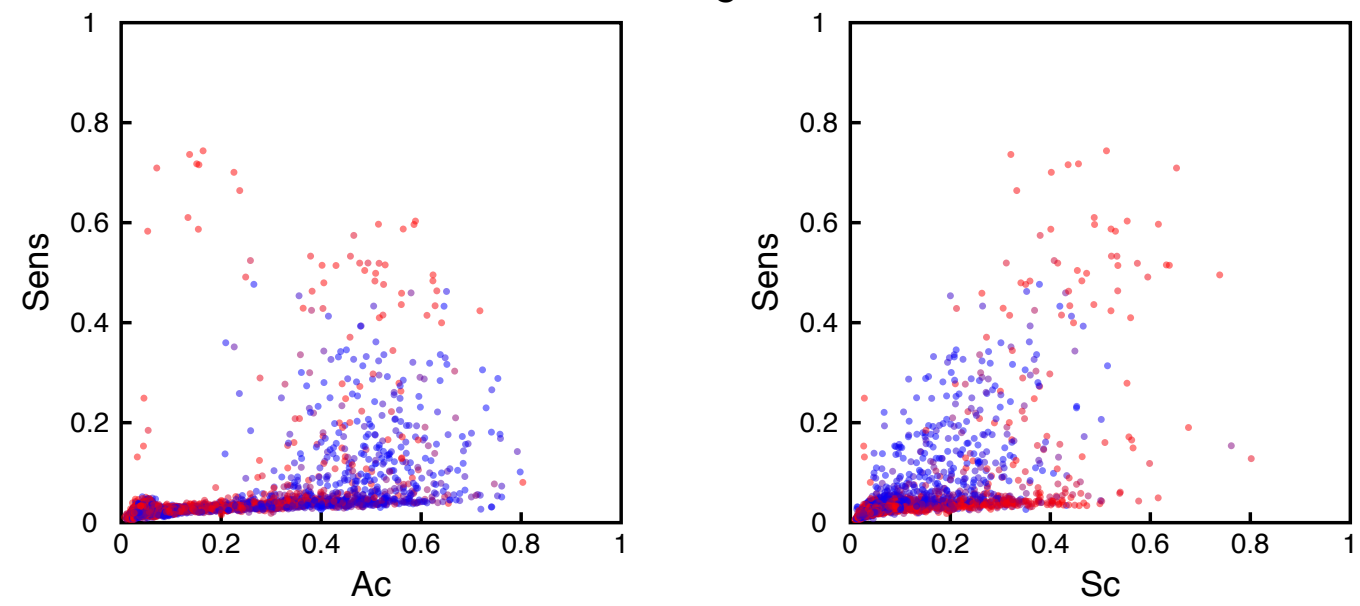

Fig. S6. Proneural activity pattern.

(A) Expression patterns of Ac (Cherry-Ac, red), Sc (GFP-Sc, green) and Sens (blue) at $8 \mathrm{~h} \mathrm{APF}$. In resolving stripes 2 and 4, Sc is expressed in fewer cells than Ac. (B) Scatter plot of Ac vs. Sens levels measured in individual cells. All cells of the notum region 
encompassing stripes 1-5 were measured. Sens expression is restricted to cells with high Ac, with the exception of emerging SOPs with highest Sens levels. (C) Scatter plot of Sc vs. Sens levels in individual cells. Two populations are seen: a branch with comparable levels of Sc and Sens (along the diagonal of the plot), and a branch of Sc-expressing cells with low Sens, corresponding to cells within resolving stripes. Cells in (B) and (C) are colored by position relative to the stripes (red: center of resolving stripes; blue: center of emerging stripes). 


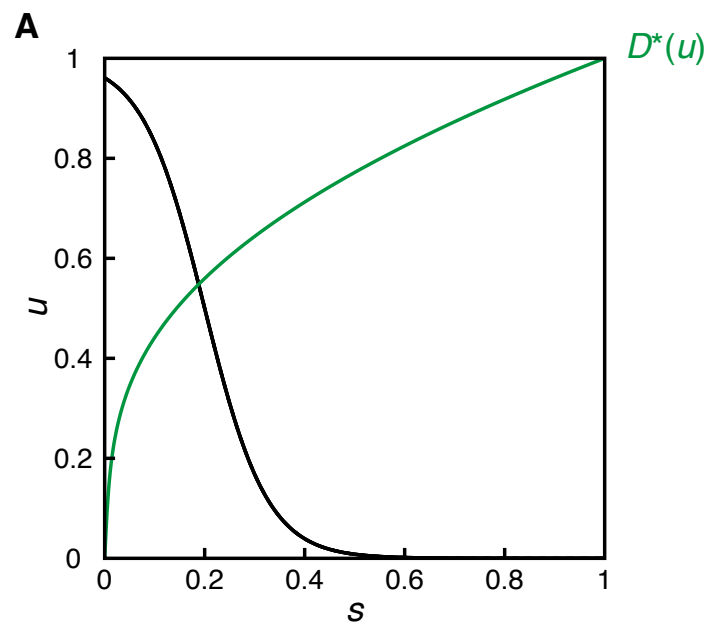

\section{B}

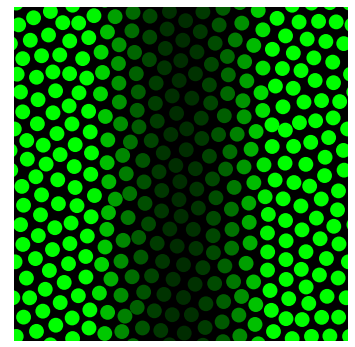

$t=0$

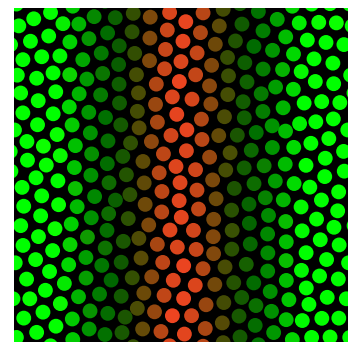

$t=1$

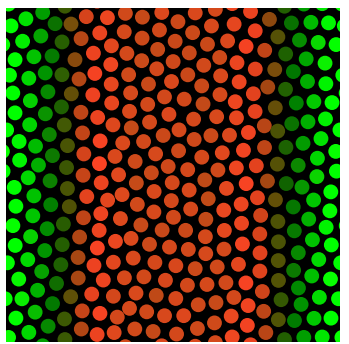

$t=2$

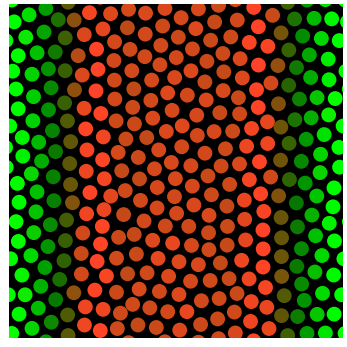

$t=5$

Fig. S7. A monostable model with moderate non-linearities fails to pattern.

(A) The response function of this model, Eq. S19, gives rise to a single, stable branch that varies smoothly with the signal $s$. The signal produced by a cell, $D^{*}(u)$, is the same as in our main model, Eqs. 10 and 11. (B) These moderate non-linearities are insufficient to destabilize a uniform steady state, and all cells remain at an intermediate level of $u$. 
A

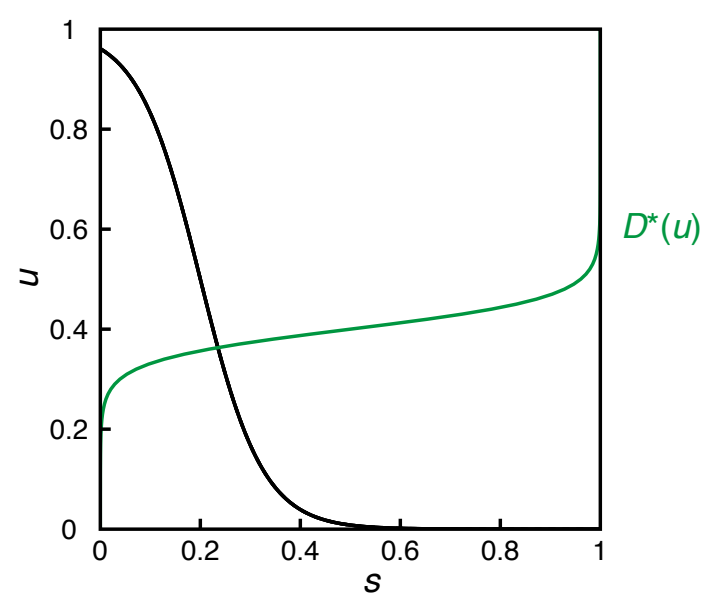

B

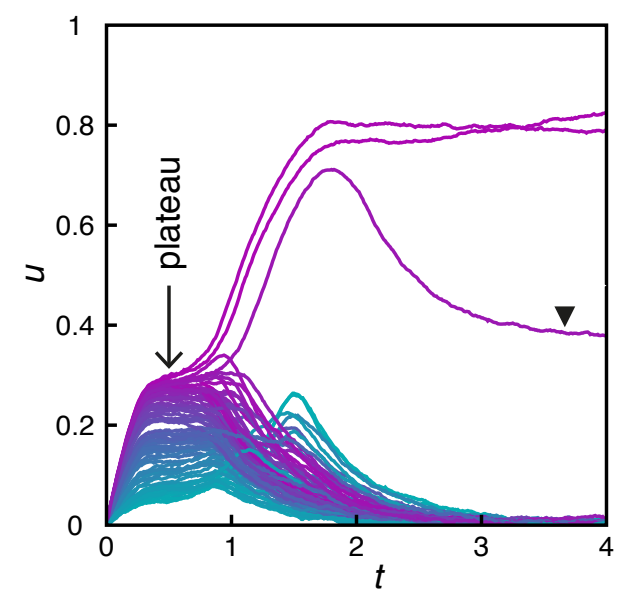

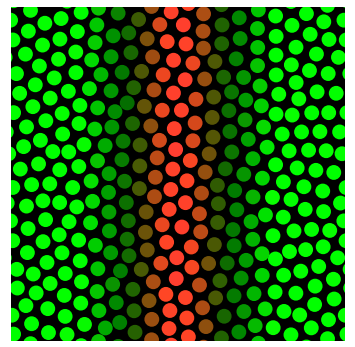

$t=.5$

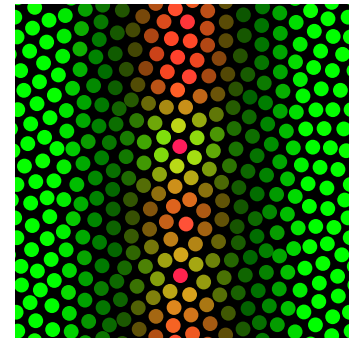

$t=1$

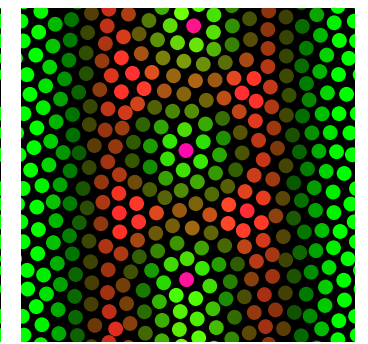

$t=1.5$

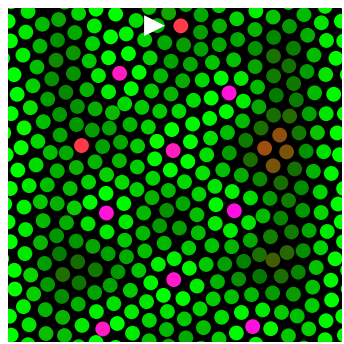

$t=5$

Fig. S8. A monostable model with a sharp onset of signaling.

(A) This model has the same, relatively smooth response curve as in fig. S7, Eq. S19, but a sharper signal-sending curve, Eq. S20. (B) Time courses of cell state $u$ in stripe 3, with color indicating position within the stripe (magenta: center; blue: sides). (C) Snapshots of the model at successive time points. This model yields a sequence of proneural stripes that resolve into isolated SOPs $(\mathrm{B}, \mathrm{C})$, but the dynamics is qualitatively different from our bistable model. In the monostable model considered here, cells in the stripe rapidly tend to a near-steady state (see the plateau in B and $\mathrm{C}, t=.5$ ), then isolated SOPs emerge as a result of small-scale fluctuations $(\mathrm{C}, t=1$ and $t=1.5)$. By contrast, stripe 3 narrows down before resolving in our main model (Fig. 5A,B). Arrowheads ( $\mathrm{B}$ and $\mathrm{C}, t=5)$ highlight a cell that remains in an intermediate state (ill-defined fate) as the pattern approaches steady state. 
A

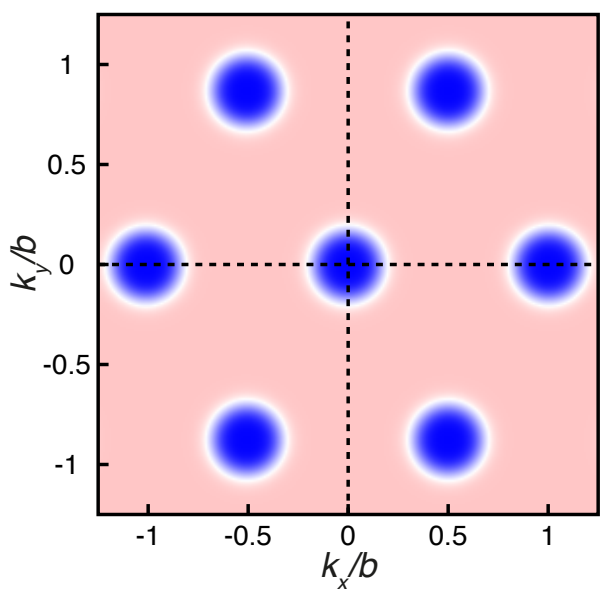

C

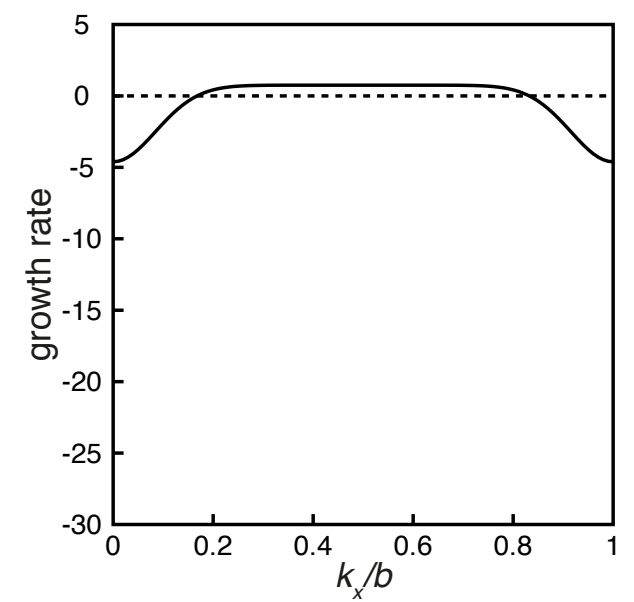

\section{E}

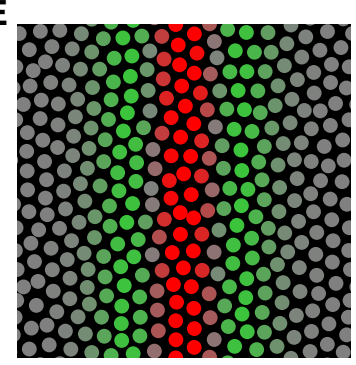

$\mathbf{F}$

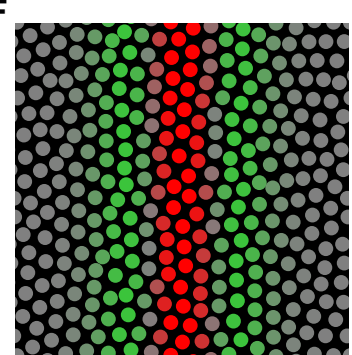

$t=0$
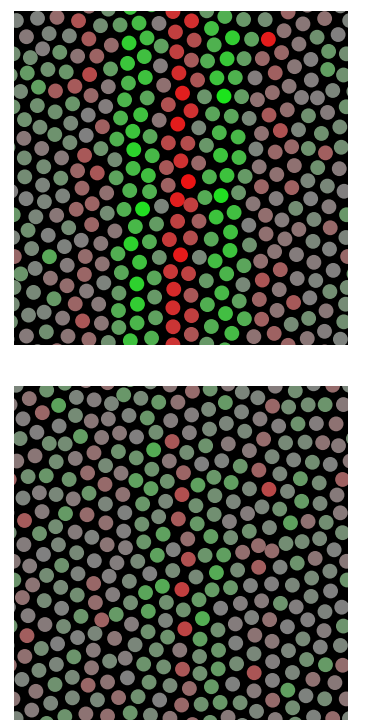

$t=.5$
B

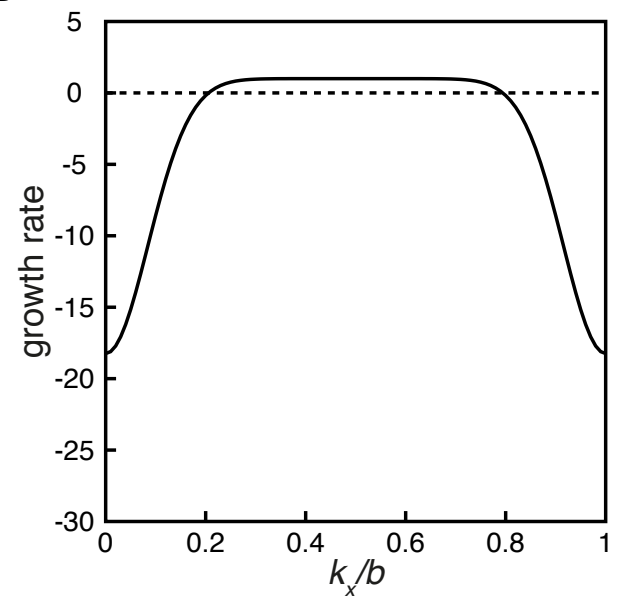

D

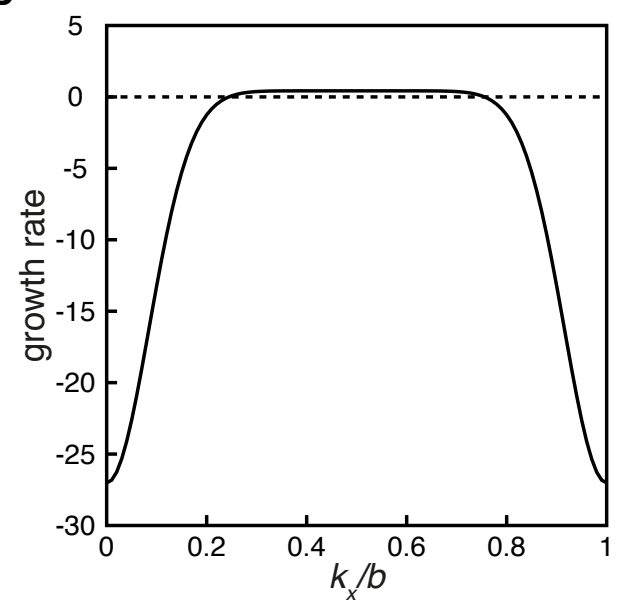

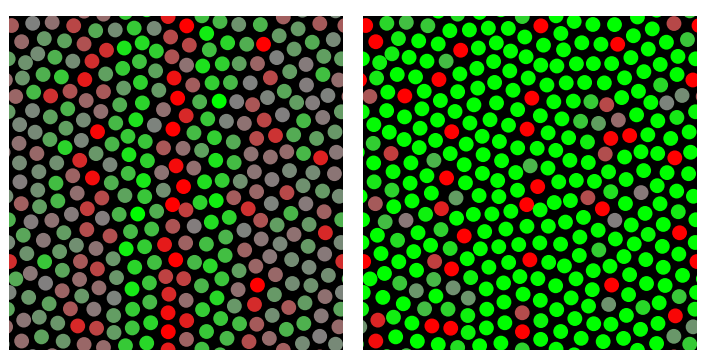

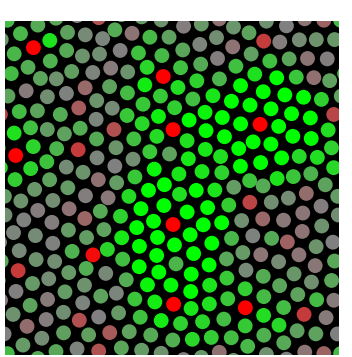

$t=1$

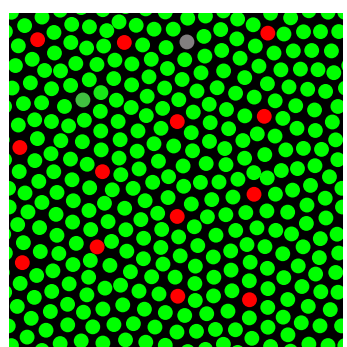

$t=2$

Fig. S9. Linear stability analysis of a bistable vs. monostable model.

The growth rate of perturbations to a uniform steady state, Eq. S28, integrates a 
contribution from cell-intrinsic dynamics and a contribution from cell-cell interactions. (A-D) show the contribution of cell-cell interactions alone, corresponding to the dynamics $d u / d t=-s$. (A) Growth rate of perturbations $-\hat{c}(\vec{k})$ as a function of the wave vector $\vec{k}=\left(k_{x}, k_{y}\right)$. Growing perturbations are in shown red, decaying perturbations in blue. (B) Section of A along the horizontal axis. Lateral inhibition tends to dampen large scale-modes (small wavenumbers) and amplify small-scale modes (large wavenumbers). $(\mathbf{C}, \mathbf{D})$ Growth rate of perturbations in our main model (C) and in the monostable model of fig. S8 (D). The monostable model has a narrower range of unstable modes (with positive growth rate) and large-scale perturbations are much more strongly damped. $(\mathbf{E}, \mathbf{F})$ Autonomous resolution of proneural stripes is explained by a bistable, not a monostable model. The two simulations were initiated with a small perturbation to a uniform, near-steady state (red: higher $u$; green: lower $u$ ), and run in the absence of extrinsic signaling. With our bistable model, a band of higher $u$ can sustain itself and narrow down before it resolves (E). In contrast, with the monostable model of fig. S8, it rapidly decays, then isolated SOPs emerge from a near-uniform background (F). 
A
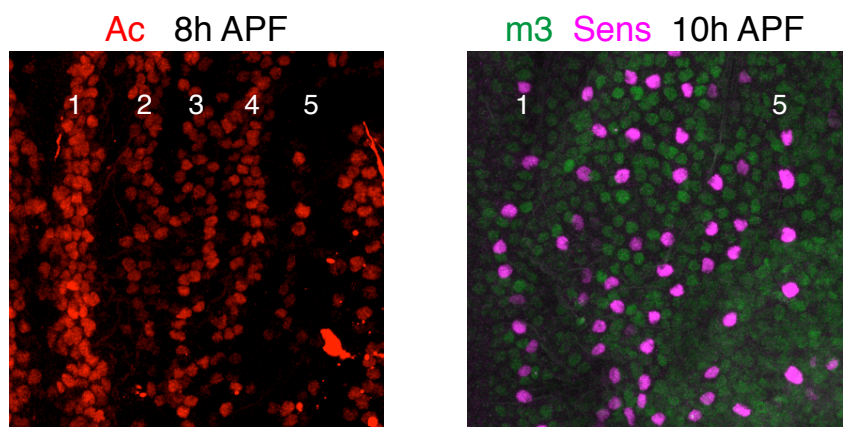

B

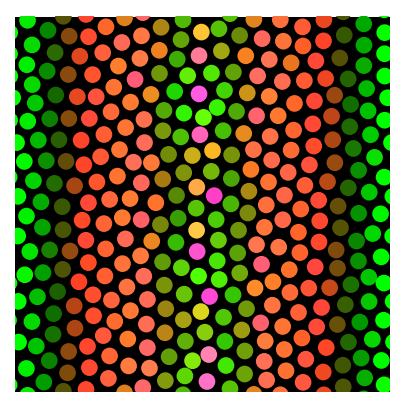

$t=2$

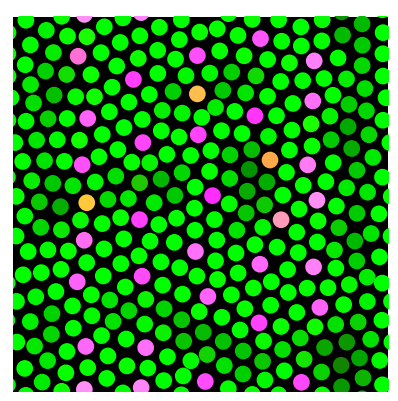

$t=5$

Fig. S10. Patterning in a sca mutant.

Reducing the range of signaling in vivo (A; sca mutant pupae) and in the model (B; $l=1.25 \lambda$ instead of $1.75 \lambda$ ) did not perturb stripe patterning (compare the pattern of Ac stripes at $8 \mathrm{~h} \mathrm{APF}$ in $s c a$ and wild-type pupae; cf. fig S2B; compare also the simulation with the pattern at $t=2$ in Fig. $2 \mathrm{E}$ ). At later stages ( $10 \mathrm{~h} \mathrm{APF}$ and $t=5)$, supernumerary SOP rows are observed. 
A

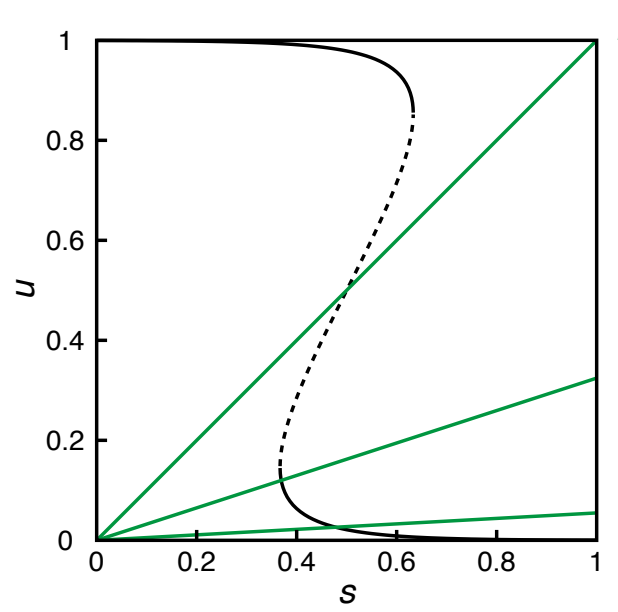

C

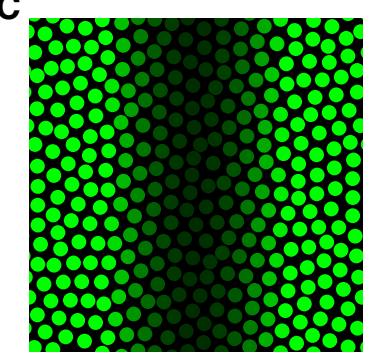

D

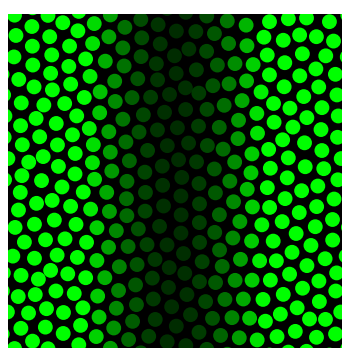

$t=0$
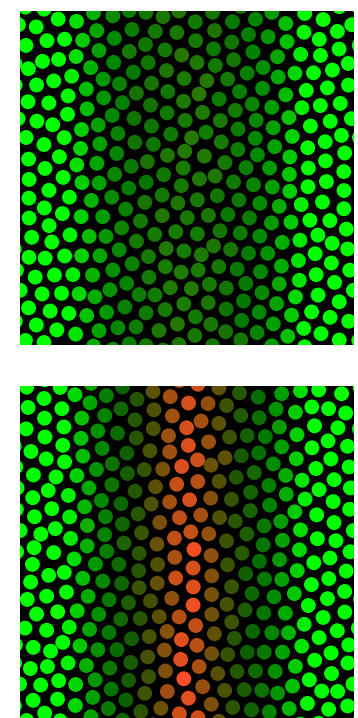

$t=1$

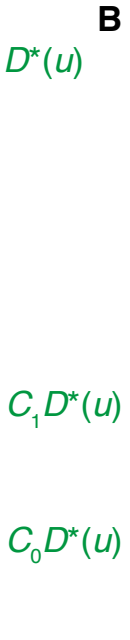

$D^{*}(u) \quad C_{1} D^{*}(u)$
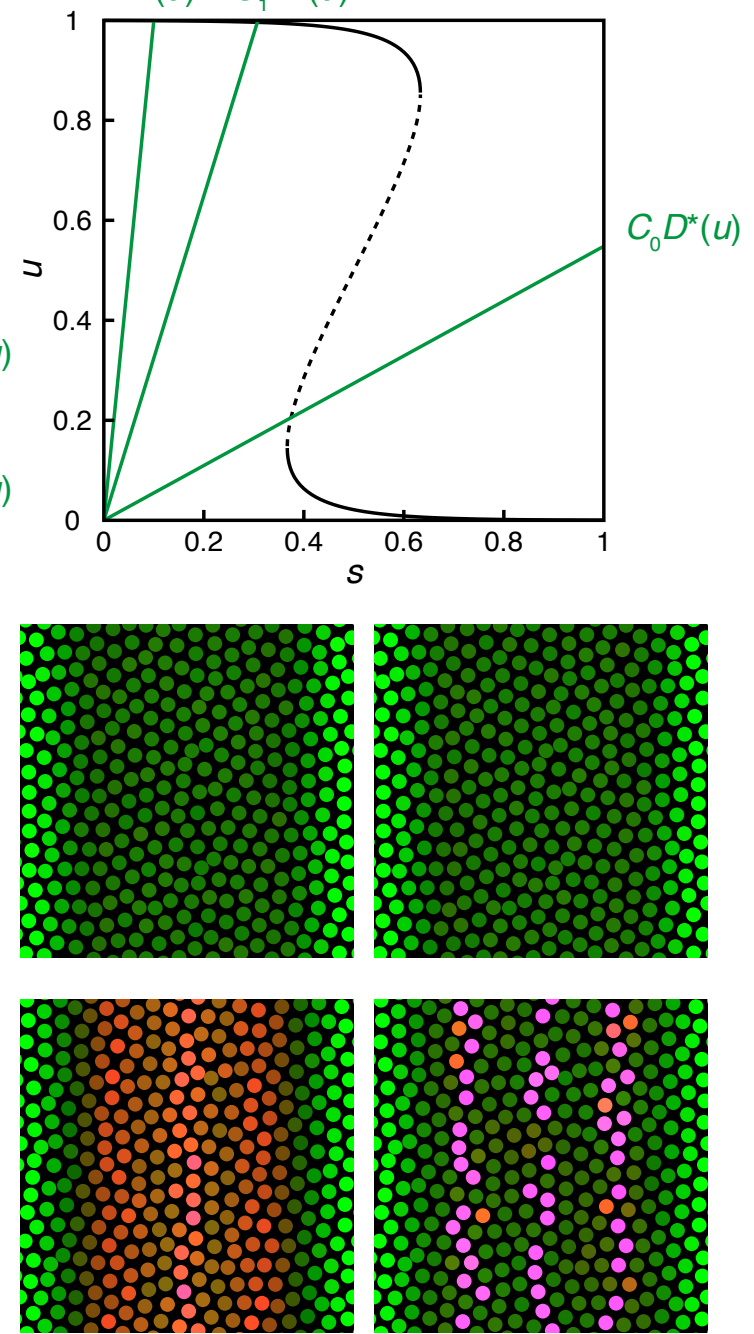

$t=2$

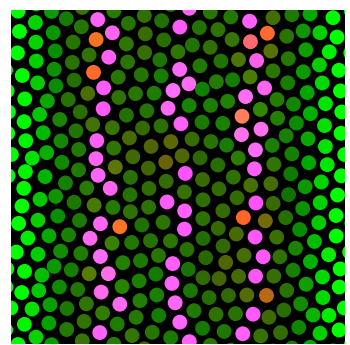

$t=5$

Fig. S11. Requirement for a nonlinear progression in signal-sending activity.

With a linear increase in the signal $D^{*}(u)$ sent by a cell as a function of its state $u$, the model cannot account for the dynamics of SOP patterning in the notum. This is illustrated by two examples, with different levels of inhibition. Top panels $(\mathbf{A}, \mathbf{B})$ show signaling activity in different configurations overlaid on the bifurcation diagram of the model as in Fig. 4B. Bottom panels $(\mathbf{C}, \mathbf{D})$ show the resulting patterning dynamics. In a first example $(\mathrm{A}, \mathrm{C})$, we take $D^{*}(u)=u$ with the same maximum signal as in our main model (Fig. 4B). In this scenario, the bottom green line (corresponding to all equivalent cells) hits the stable, low- $u$ branch (A). Mutual inhibition is not sufficient to destabilize the corresponding uniform steady state and all cells remain in a state with intermediate signal and low $u(\mathrm{C})$. For a group of cells to start progressing towards the SOP fate (as in a proneural stripe), the bottom green curve must intersect the steady-state curve above its turning point. This requires a much weaker mutual inhibition, e.g. $D^{*}(u)=.1 u(\mathrm{~B})$. In this second scenario, proneural stripes cannot resolve into isolated SOPs (D). 
A

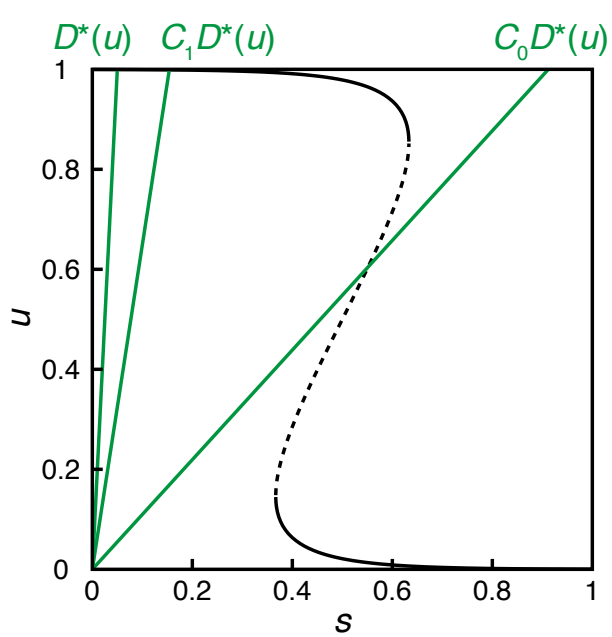

C

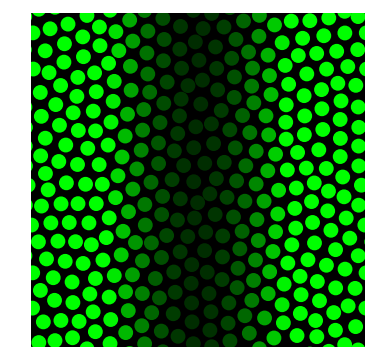

D

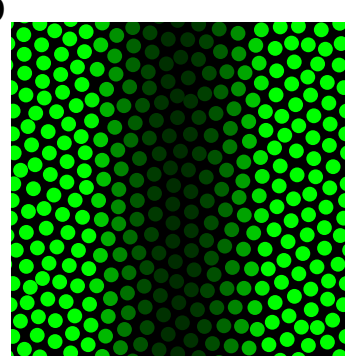

$t=0$
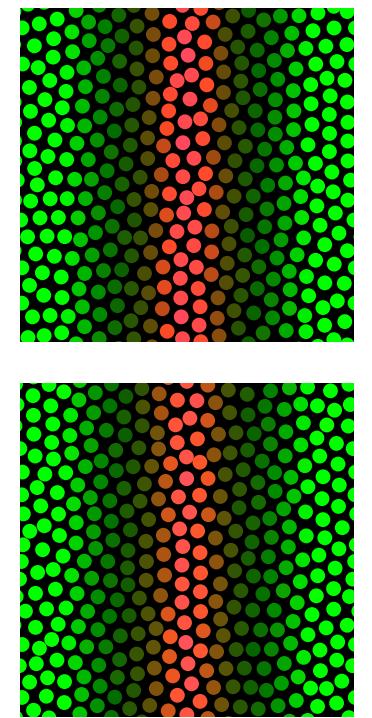

$t=1$
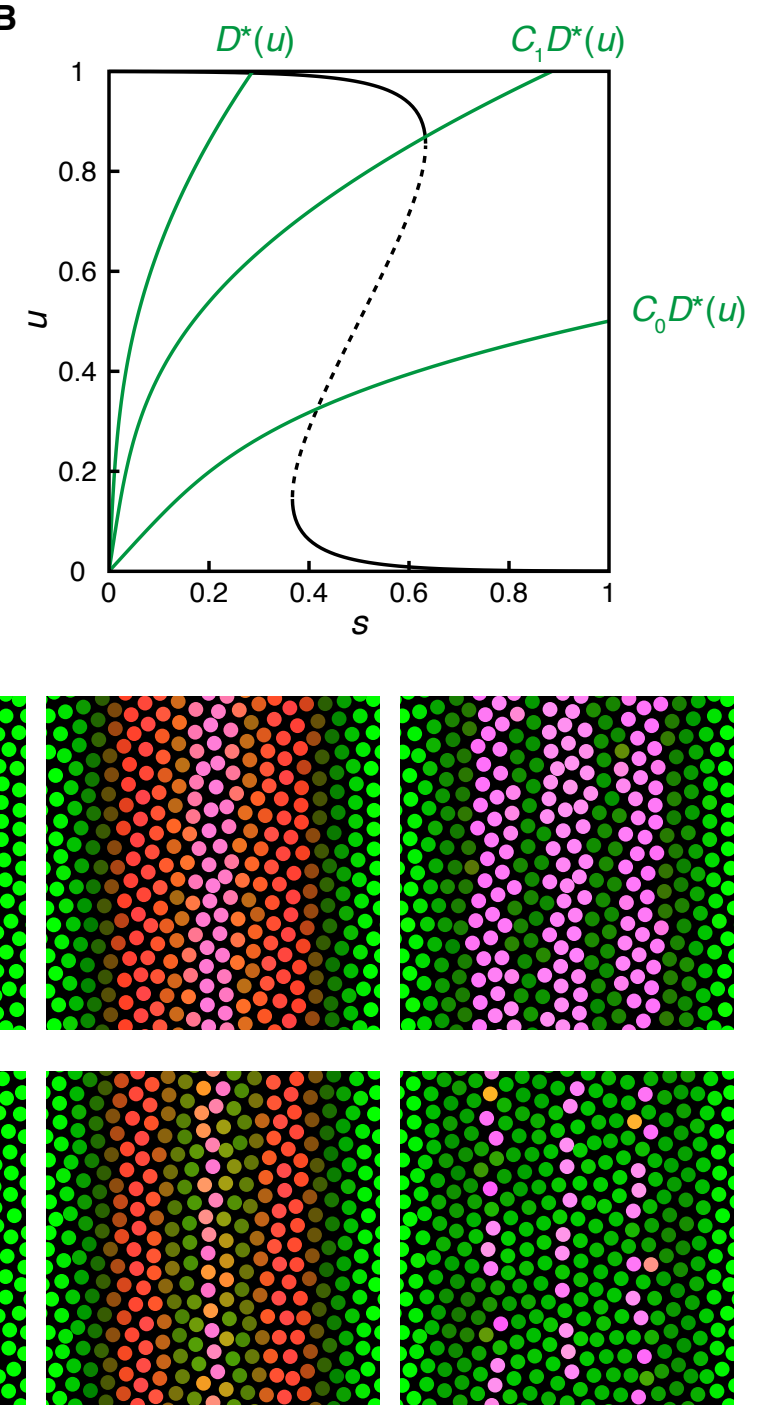

$t=2$

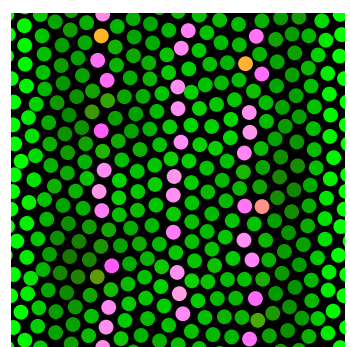

$t=5$

Fig. S12. Model dynamics with reduced enhancement of Dl activity in SOPs.

$(\mathbf{A}, \mathbf{B})$ Signaling activity overlaid on the bifurcation diagram of the model (cf. Fig. 4A) with no enhancement (A; $a_{1}=0$ in Eq. 11) or a weaker enhancement of Dl activity in SOPs $\left(\mathrm{B} ; a_{1}=\left(1-a_{0}\right) / 4\right.$ in Eq. 11$)$. (C,D) Snapshots of the corresponding models at successive time points. With no enhancement $(\mathrm{C})$, the green line $s=C_{0} D^{*}(u)$ still hits the unstable branch of the bifurcation diagram, implying that a uniform steady state is unstable. But mutual inhibition within a row or pair of equivalent cells $\left(s=C_{1} D^{*}(u)\right.$ and $\left.s^{*} D^{*}(u)\right)$ is much lower than the threshold for inhibition of the SOP fate. Consistent with this, proneural stripes can form, but they fail to resolve, yielding continuous bands of SOPs. In experiments, loss of neur function has a more moderate effect (Fig. 6D'). This suggests a neur-independent element of non-linearity, e.g. in the increase of D1 expression in SOPs. A similar outcome is obtained with a partial reduction of Dl activity enhancement in SOPs (B): the final pattern shows an alternation of SOPs and epidermal cells (D). 
$t=2$
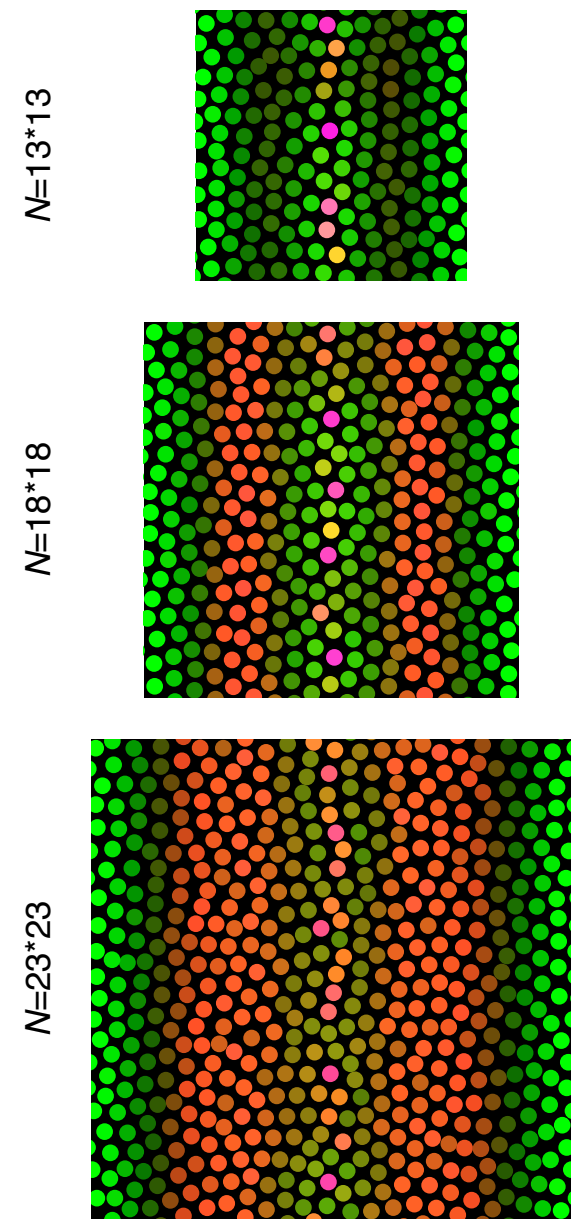

$t=10$
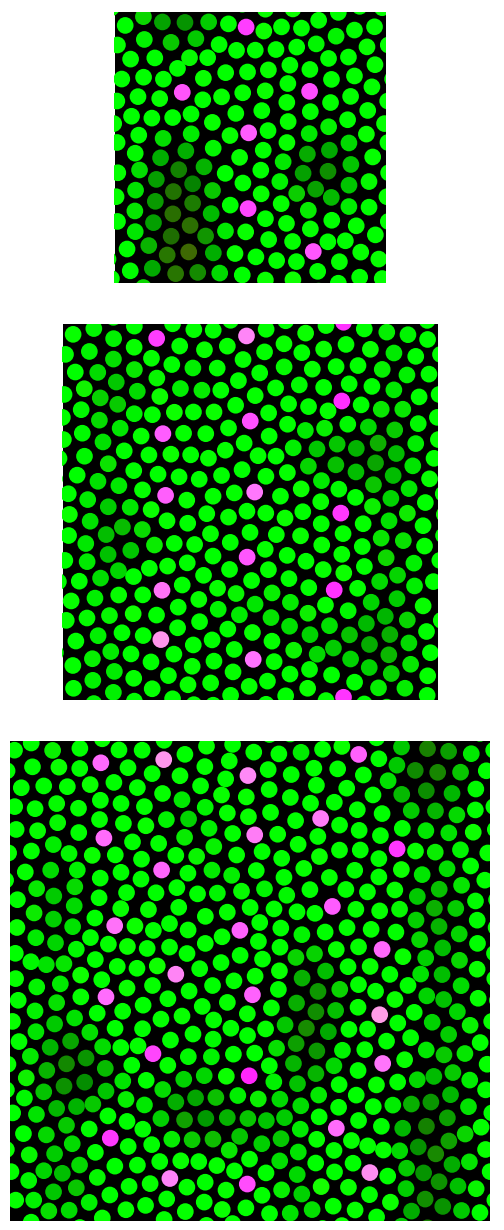

\section{Fig. $S$ 13. Effect of tissue size on patterning in the model.}

Different tissue sizes are modeled by changing the number of cells $\mathrm{N}$ in the unit simulation box. All other parameters are left unchanged, thus the signaling range relative to the typical cell-cell distance is fixed, and the profile of the initial signaling gradient scales with tissue size. The middle row corresponds to the number of cells in our main model. In a small enough tissue, SOP rows are lost, while in a large enough tissue, supernumerary rows form. Moderate size variations are shown here, yielding partial loss and gain of rows. 
Table S1. Fly genotypes.

\begin{tabular}{|c|c|}
\hline$w ; \mathrm{M}[\mathrm{GFP}-\mathrm{m} 3] 51 \mathrm{D} /+$ & Fig. 1B,G \\
\hline$w ; \mathrm{M}[\mathrm{GFP}-\mathrm{m} 3] 51 \mathrm{D} /+; \mathrm{PB}[\mathrm{Cherry}-\mathrm{Ac}] \mathrm{VK} 33 /+$ & $\begin{array}{l}\text { Fig. } 1 \mathrm{C}, \mathrm{E}, \mathrm{F} \\
\text { Fig. } 3 \mathrm{~A} \\
\text { fig. S2B,C }\end{array}$ \\
\hline scute $^{\text {GFP }} / \mathrm{Y} ;$; PB[Cherry-Ac]VK33 / + & $\begin{array}{l}\text { Fig. 1D } \\
\text { fig. S1B } \\
\text { fig. S2A } \\
\text { fig. S6 }\end{array}$ \\
\hline $\begin{array}{l}\text { w P[Ubx-flp] ; M[GFP-m3]51D / + ; FRT82B D } l^{\text {rev } 10} / \text { FRT82B P[ubi- } \\
\text { mRFPnls }]\end{array}$ & Fig. $1 \mathrm{H}$ \\
\hline scute $^{G F P} / \mathrm{Y} ; ; \mathrm{P}[\mathrm{H} 2 \mathrm{Av}-\mathrm{mRFP}] /+$ & $\begin{array}{l}\text { Fig. 3B } \\
\text { Fig. 5C,D } \\
\text { movie S2 }\end{array}$ \\
\hline $\begin{array}{l}w N^{55 e 11} \text { FRT19A / P[hs-flp]12 P[ubi-mRFPnls] FRT19A ; ; PB[GFP- } \\
\text { Ac]VK33 / + }\end{array}$ & Fig. 6A' \\
\hline$w ; \mathrm{M}[\mathrm{GFP}-\mathrm{m} 3] 51 \mathrm{D} /+; \mathrm{PB}[$ Cherry-Ac]VK33 / FRT82B Dlev10 & Fig. 6B' \\
\hline$w ; s c a^{B P 2} / s c a^{l} ; \mathrm{PB}[\mathrm{GFP}-\mathrm{Ac}] \mathrm{VK} 33$ / P[H2Av-mRFP] & Fig. $6 \mathrm{C}^{\prime}$ \\
\hline $\begin{array}{l}w \text { P[Ubx-flp] P[pneur-eqFP670nls]attP3 ; ; FRT82B neur }{ }^{I F 5} \text { / FRT82B } \\
\text { P[ubi-mRFPnls] }\end{array}$ & Fig. 6D' \\
\hline$w ; ;$ Delta $^{G F P}$ & fig. S3 \\
\hline$w ;$; neur ${ }^{P \text { Gal4 }} \mathrm{P}[\mathrm{UAS}-\mathrm{GFP}-\mathrm{Moe}] /+$ & fig. S4 \\
\hline$w ; s c a^{B P 2}$ M[GFP-m3]51D / sca $a^{l}$ PB[Cherry-Ac]VK33 / + & fig. S10A \\
\hline $\mathrm{w} ; \mathrm{P}[\mathrm{H} 2 \mathrm{Av}-\mathrm{mRFP}] /+; \mathrm{PB}[\mathrm{GFP}-\mathrm{Ac}] \mathrm{VK} 33 /+$ & movie S1 \\
\hline$w ; \mathrm{P}[\mathrm{H} 2 \mathrm{Av}-\mathrm{mRFP}] /+;$ Delta $^{\text {GFP }} /+$ & movie S3 \\
\hline$w ; \mathrm{M}[\mathrm{GFP}-\mathrm{m} 3] 51 \mathrm{D} / \mathrm{P}[\mathrm{H} 2 \mathrm{Av}-\mathrm{mRFP}]$ & movie S4 \\
\hline
\end{tabular}


Table S2. Parameters values.

This table lists the parameter values used for the different models and the changes made to describe perturbations. The signaling range $l$ is defined in units of the typical cell-cell distance $\lambda$, defined by $n \lambda^{2}=1$, where $n$ is the density of cells per unit area.

\begin{tabular}{|c|c|}
\hline Description & Parameter values \\
\hline $\begin{array}{l}\text { Main model } \\
\text { Eqs. } 4-12 \\
\text { Figs. } 2,4,5 \mathrm{~A}, \mathrm{~B} \text {, fig. S9C,E, movie S5 }\end{array}$ & $\begin{array}{l}l=1.75 \lambda, \tau=1 / 2, D=5.10^{-5}, a_{0}=.05, a_{1}=1-a_{0}, \\
S_{0}=2, L=.2, \tau_{g}=1, N=324\end{array}$ \\
\hline \multicolumn{2}{|c|}{ Perturbations } \\
\hline $\begin{array}{l}\text { Notch mutant clone } \\
\text { Fig. 6A }\end{array}$ & $s=0$ for cells within the clone \\
\hline $\begin{array}{l}D l+/- \\
\text { Fig. } 6 \mathrm{~B} \\
\end{array}$ & $S_{0}=1$ and $D(u)=u / 2$ \\
\hline $\begin{array}{l}\text { sca mutant } \\
\text { Fig. } 6 \mathrm{C} \text {, fig. S10B }\end{array}$ & $l=1.25 \lambda$ \\
\hline $\begin{array}{l}\text { Linear signal-sending curve } \\
\text { fig. S11 }\end{array}$ & $D^{*}(u)=u, D^{*}(u)=.1 u$ \\
\hline $\begin{array}{l}\text { neur mutant } \\
\text { Fig. } 6 \mathrm{D} \text {, fig. S12A,C }\end{array}$ & $a_{1}=0$ \\
\hline $\begin{array}{l}\text { Partial reduction in Dl activity } \\
\text { fig. } \mathrm{S} 11 \mathrm{~B}, \mathrm{D}\end{array}$ & $a_{1}=\left(1-a_{0}\right) / 4$ \\
\hline $\begin{array}{l}\text { Variations in tissue size } \\
\text { fig. S13 }\end{array}$ & $N=169,324,529$ \\
\hline \multicolumn{2}{|c|}{ Alternative models } \\
\hline $\begin{array}{l}\text { Model with cis-inhibition } \\
\text { Eqs. S7, S10-15 } \\
\text { Eqs. 5, 7, 9, } 11 \text { from main model } \\
\text { fig. S5 }\end{array}$ & $\begin{array}{l}\beta_{N}=2, \gamma_{N}=\gamma_{D}=1, k_{c}=100, k_{t}=1, \tau=1 / 3 \\
\text { Other parameters as in main model: } \\
l=1.75 \lambda, D=5.10^{-5}, a_{0}=.05, a_{1}=1-a_{0}, S_{0}=2 \\
L=.2, \tau_{g}=1, N=324\end{array}$ \\
\hline $\begin{array}{l}\text { Monostable model with moderate } \\
\text { nonlinearities } \\
\text { Eq. S19 } \\
\text { Eqs. 5, 7-12 from main model } \\
\text { fig. S7 }\end{array}$ & $\begin{array}{l}c=4, s_{c}=.2 \\
\text { Other parameters as in main model: } \\
l=1.75 \lambda, \tau=1 / 2, D=5.10^{-5}, a_{0}=.05, a_{1}=1-a_{0}, \\
S_{0}=2, L=.2, \tau_{g}=1, N=324\end{array}$ \\
\hline $\begin{array}{l}\text { Monostable model with sharp onset of } \\
\text { signaling } \\
\text { Eqs. S19, S20 } \\
\text { Eqs. 5, 7-9, } 12 \text { from main model } \\
\text { fig. S8, fig. S12F }\end{array}$ & $\begin{array}{l}c=4, s_{c}=.2 \\
u_{c}=.4, k=8 \\
\text { Other parameters as in main model: } \\
l=1.75 \lambda, \tau=1 / 2, D=5.10^{-5}, S_{0}=2, L=.2, \\
\tau_{g}=1, N=324\end{array}$ \\
\hline
\end{tabular}




\section{Movie S1. Live imaging of Ac expression.}

This movie shows an interval of $1.8 \mathrm{~h}$, starting at $5-6 \mathrm{~h}$ APF. The midline is near the center, anterior is up, and nuclei are labeled in red (field of view $\approx 350 \times 350 \mu \mathrm{m}$ ). Ac (GFP-Ac, green) is first expressed in stripes 1,3, and 5, then stripes 2 and 4 intercalate between them (cf. Fig 1C-F). The movie ends as stripes 2 and 4 begin to resolve.

\section{Movie S2. Live imaging of Sc expression.}

This movie shows an interval of $4 \mathrm{~h}$, starting at 5-6 h APF. The midline is near the center, anterior is up, and nuclei are labeled in red. Sc (GFP-Sc, green) is initially expressed in stripe 5, and more faintly in stripes 1 and 3 on each side (cf. Fig 1D). These first stripes later become refined and stripes 2 and 4 intercalate between them. As the movie ends, stripes 2 and 4 are partially resolved.

\section{Movie S3. Live imaging of Dl expression.}

This movie shows an interval of $2.8 \mathrm{~h}$, starting at $5-6 \mathrm{~h}$ APF. The midline is to the left, anterior is up, and nuclei are labeled in red. As the movie begins, the two heminota are migrating towards the midline (suggesting an earlier stage than movies S1 and S2). D1 (Dl-GFP, green) is initially expressed at the locations of proneural stripes 1 and 5, and expression in stripe 3 emerges as the heminota meet and fuse (cf. fig. S3). Stripe 5 gradually narrows from a broad stripe to a narrow file of cells (stripes 1 and 3 are less visible because of tissue folding and uneven contrast).

\section{Movie S4. Live imaging of Notch activity.}

This movie shows an interval of $2.3 \mathrm{~h}$ starting at 5-6 h APF. As in movies S1 and S2, the midline is near the center, anterior is up, and nuclei are labeled in red. Initially, Notch (GFP-m3, green) is activated in a series of bands along the sides of the future stripes 1 and 5 (cf. Fig 1C). Over time, the range of Notch activity around stripes 1 and 5 recedes; a pair of GFP-m3 bands closes in on stripe 5. At the same time, GFP-m3 is expressed in stripe 3, showing the onset of mutual inhibition within the stripe. As the movie ends, isolated cells with low Notch activity, corresponding to emerging SOPs, are visible within the stripes.

\section{Movie S5. Dynamics of the model.}

This movie shows the same simulation as Fig. 2E, with the same color code (green: inhibitory signal; red/magenta: cell state). As the initial gradient of inhibition recedes, stripes 2-4 emerge and resolve into SOP rows. 